\title{
Methodische Implikationen verschiedener theoretischer Resilienzmodelle
}

\author{
Otto Bodi-Fernandez $\cdot$ Karina Fernandez
}

Online publiziert: 26. Mai 2020

(C) Der/die Autor(en) 2020

Zusammenfassung Der Beitrag befasst sich mit der Frage nach Möglichkeiten der Operationalisierung des Phänomens, das mit dem Begriff Resilienz beschrieben wird. In der bisherigen Forschung wurden verschiedenste Strategien verfolgt, die ebenso vielseitig sind wie die theoretischen Konzepte und Definitionen des Begriffs. Während etwa viele Arbeiten Resilienz als ein relationales Konstrukt verstehen, das eine bestimmte Beziehung von Risiko- und Schutzfaktoren beschreibt, fassen andere Arbeiten Resilienz als eine Persönlichkeitseigenschaft auf, die auch mehr oder weniger direkt gemessen werden kann. Beide Auffassungen erscheinen methodisch nicht miteinander vereinbar. Des Weiteren weisen sie ihre spezifischen Stärken und Schwächen auf. Anhand der Daten eines im Jahr 2013 durchgeführten Kindersurveys will der Beitrag aufzeigen, welche Implikationen sich aus Resilienzkonzepten für empirisches Material ergeben und welche theoretischen Resilienzmodelle in unterschiedlichen Kontexten empirisch beobachtbar sind. Es wird aufgezeigt, dass bei eventuell nachweisbaren Resilienz-Effekten additive Effekte, Mediatoreffekte und Moderatoreffekte differenziert werden sollten. Wenngleich Moderatoreffekte den gängigen Auffassungen von Resilienz am nächsten kommen, bleibt es letztendlich eine analytisch-definitorische Frage, ab wann von Resilienz gesprochen werden kann.

Schlüsselwörter Resilienz $\cdot$ Kinder $\cdot$ Kindheitsforschung $\cdot$ Methodik

\footnotetext{
O. Bodi-Fernandez $(\bowtie)$

Center for Social Research, Universität Graz, Universitätsstraße 15/4G, 8010 Graz, Österreich

E-Mail: otto.bodi@uni-graz.at

K. Fernandez

Institut für Educational Governance, Pädagogische Hochschule Steiermark, Ortweinplatz 1/II, 8010 Graz, Österreich

E-Mail: karina.fernandez@phst.at
} 


\title{
Methodological implications of different theoretical models of resilience
}

\begin{abstract}
The paper deals with the question of possible operationalizations of the phenomenon, which is described by the term resilience. Previous research has pursued a variety of strategies that are as diverse as the theoretical concepts and definitions of the term. While in many works resilience is understood as a relational construct that describes a particular relationship between risk and protective factors, other works consider resilience as a personality trait that can be measured more or less directly. Both views seem methodically incompatible. Furthermore, they have their specific strengths and weaknesses. On the basis of the data of a children's survey carried out in 2013, the article aims to show what implications arise from resilience concepts for empirical material and which theoretical models of resilience can be empirically observed in different contexts. It is shown that additive, mediating and moderating effects should be differentiated if any resilience effects can be proven. Although moderating effects come closest to common perceptions of resilience, it remains an analytic-definitional question when to speak of resilience.
\end{abstract}

Keywords Resilience · Children · Childhood research · Methodology

\section{Einleitung}

Das Konzept der Resilienz erfreut sich seit Jahren sowohl in der wissenschaftlichen als auch gesellschaftspolitischen Diskussion großer Popularität (vgl. Bonß 2015, S. 159). Nicht nur wird Resilienz in verschiedenen Disziplinen wie der Psychologie, der Humangeografie oder den Ingenieurswissenschaften diskutiert, der Begriff ist mittlerweile im Alltagsverständnis angekommen und wird auch in populärwissenschaftlichen Magazinen und Ratgeberliteratur aufgegriffen, deren Seriosität aber eher zweifelhalft bleibt. Die oft unkritische Rezeption vor allem im Bereich der mikrosoziologischen oder psychologischen Kindheitsforschung, auf die in diesem Beitrag fokussiert wird, führte laut Fingerle dazu, ,dass der Resilienzbegriff zu einem Modekonzept wurde, zu einem nahezu beliebig verwendbaren Marketingvehikel für Trainingsprogramme“ (Fingerle 2007, S. 299). Der Reiz des Resilienzkonzepts für die Kindheitsforschung liegt wohl darin begründet, dass sich mit ihm ein Perspektivenwechsel in Forschung und Praxis vollzog. Durch die vielfältigen Studienergebnisse zu den negativen Auswirkungen eines Lebens in Ungleichheit und Armutslagen hatte ein gewisser Pessimismus in die ,helfenden“ Professionen wie Sozialpädagogik Einzug gehalten. Mit dem Resilienzkonzept erfolgte eine Verschiebung von einer defizitorientierten Haltung hin zu einer ressourcenorientierten Sichtweise (vgl. Gabriel 2005, S. 208 f.).

Trotz der „Allgegenwart“ des Resilienzbegriffs (vgl. Friedrich 2012, S. 1) bleibt dieser in der Forschung vage und schwer zu operationalisieren (Promberger et al. 2015, S. 271). Empirische Studien zu Resilienz unterscheiden sich in der Auffassung, was unter Resilienz verstanden wird, wie sie messbar gemacht wird und mit welchen methodischen Instrumenten die Erhebungen durchgeführt werden. Der vorliegende Beitrag befasst sich mit der Frage nach der empirischen Erfassung des 
facettenreichen Phänomens Resilienz. In der bisherigen Forschung wurden dazu verschiedenste Strategien verfolgt, die ebenso vielseitig sind wie die theoretischen Konzepte und Definitionen des Begriffs. In dieser Arbeit wird anhand der Daten einer 2013 durchgeführten Studie zum Thema Lebenslagen und Wohlbefinden von Kindern gezeigt, welche Implikationen sich aus Resilienzkonzepten für empirisches Material ergeben und welche theoretischen Resilienzmodelle in unterschiedlichen Bereichen empirisch beobachtbar sind. Ziel der empirischen Analysen in diesem Beitrag ist erster Linie, die verschiedenen Modelle an inhaltlichen Beispielen zu veranschaulichen und damit einen methodischen Beitrag zur empirischen Operationalisierung von Resilienz und der Entwicklung theoretisch begründeter und empirisch erfassbarer Resilienzmodelle zu leisten. Dabei wird gezeigt, dass bei etwaig nachweisbaren Resilienzeffekten zwischen additiven Effekten, Mediatoreffekten und Moderatoreffekten differenziert werden sollte, wobei Moderatoreffekte den gängigen Beschreibungen des Resilienzbegriffes am nächsten kommen.

\section{Das Konzept der Resilienz}

In ihrem Übersichtswerk über Resilienz bei Kindern bezeichnet Wustmann (2004) Resilienz als ,psychische Widerstandsfähigkeit von Kindern gegenüber biologischen, psychologischen und psychosozialen Entwicklungsrisiken“ (Wustmann 2004, S. 18). Kurz gesagt gehe es dabei um ,die Fähigkeit, sich von einer schwierigen Lebenssituation nicht ,unterkriegen zu lassen“ bzw. ,nicht daran zu zerbrechen““ (Wustmann 2004, S. 18). Kinder, die unter Bedingungen wie chronischer Armut oder niedrigem gesellschaftlichen Status aufwachsen, sind damit einhergehend einem erhöhten Maß an Entwicklungsrisiken ausgesetzt. Diese entfalten bei vielen Kindern ihre Wirkung und führen beispielsweise zu psychischen Belastungen oder eingeschränktem Wohlbefinden. Resiliente Kinder wachsen zwar mit denselben Entwicklungsrisiken auf, können diese Belastungen jedoch bewältigen, ohne Schaden zu nehmen (vgl. Wustmann 2005; Zander 2011). In engem Zusammenhang steht der Begriff der Resilienz mit dem Konzept der Vulnerabilität. ${ }^{1}$ „,Vulnerabilität kennzeichnet die Verwundbarkeit, Verletzbarkeit oder Empfindlichkeit einer Person gegenüber äußeren (ungünstigen) Einflussfaktoren - also eine erhöhte Bereitschaft, psychische Erkrankungen zu entwickeln“ (Wustmann 2004, S. 22). Entscheidend ist dabei nicht nur ein positives oder negatives Entwicklungsergebnis. Vielmehr ist das Resilienzkonzept an zwei wesentliche Bedingungen geknüpft: Erstens muss eine signifikante Bedrohung, also eine potenzielle Verwundbarkeit, für die kindliche Entwicklung vorliegen und zweitens eine erfolgreiche Bewältigung dieser belastenden Lebensumstände erfolgen (vgl. Wustmann 2004, S. 18, 2005, S. 192). Demzufolge können nur Kinder als resilient angesehen werden, die sich trotz belastender Lebensumstän-

\footnotetext{
1 Sowohl zum Konzept der Resilienz als auch der Vulnerabilität wird in unterschiedlichen Disziplinen ein reger Diskurs geführt, wobei sich die Konzeptionen zum Teil voneinander unterscheiden (für einen Überblick über Resilienz- und Vulnerabilitätskonzeptionen in unterschiedlichen Disziplinen siehe Bürkner 2010 oder Keck und Sakdapolrak 2013). In diesem Beitrag wird auf die in der psychologisch orientierten Kindheitsforschung gebräuchliche Form zurückgegriffen.
} 
de erstaunlich positiv entwickeln, im Vergleich zu Kindern, die unter den gleichen Umständen psychische Beeinträchtigungen aufweisen. In ähnlicher Weise charakterisiert Masten (2001) Resilienzphänomene als ,good outcomes in spite of serious threats to adaption or development" (Masten 2001, S. 228) und betont, dass demnach Individuen nicht als resilient betrachtet werden können, wenn sie nie einem erhöhten Entwicklungsrisiko ausgesetzt waren. Dieses in der Forschung weitgehend geteilte Verständnis von Resilienz hat zur Konsequenz, dass Resilienz nicht als ein individuelles Persönlichkeitsmerkmal verstanden werden kann (vgl. Wustmann 2004, S. 28; Zander 2008, S. 19). Vielmehr beschreibt Resilienz den ,Prozess, die Fähigkeit oder das Ergebnis erfolgreicher Adaptation angesichts herausfordernder oder bedrohender Umstände im Sinne inneren Wohlbefindens und/oder effektiver Austauschbeziehungen mit der Umwelt“ (Masten et al. 1990, S. 465). Auch wenn die Definition den Begriff der psychischen Widerstandsfähigkeit präzisiert, legt sie nicht fest, ob Resilienz Prozess, Fähigkeit oder Ergebnis beschreibt. Beschrieben wird eine potenzielle Größe, eine „Kapazität, die im Verlauf der Entwicklung im Kontext der Kind-Umwelt-Interaktion erworben wird“ (Wustmann 2004, S. 28). Wustmann charakterisiert Resilienz weiter als variabel, situationsspezifisch und multidimensional. So kann sich die Fähigkeit, Risikobedingungen erfolgreich zu bewältigen, in der Entwicklung eines Kindes über Zeit und Situationen sehr verändern. Auch kann ein Kind in einem Bereich wie beispielsweise der schulischen Leistung resilient, hingegen in anderen Bereichen wie etwa der sozialen Kontakte und Beziehungen nicht resilient sein (vgl. Wustmann 2005, S. 194). Die Multidimensionalität des Resilienzbegriffs lässt somit unterschiedlichste Kriterien für Resilienz zu (vgl. Lösel und Bender 2007, S. 60). Durch das Fehlen einer einheitlichen Terminologie und einheitlicher methodischer Zugänge bleibt zunächst unklar, wann ein Verhalten bzw. eine Anpassungsleistung als resilient angesehen wird. In einigen Fällen wird Resilienz als Abwesenheit psychischer Störungen definiert, in anderen als Kompetenzkriterien, die sich an der Erfüllung der altersspezifischen Entwicklungsaufgaben festmachen lassen, und wieder andere ForscherInnen sehen das „blanke Überleben“ eines Kindes als resilient an (vgl. Wustmann 2005, S. 202). Entsprechend heterogen sind auch die Forschungsergebnisse je nachdem, welche Resilienzkriterien herangezogen werden. Da es keine Patentlösung für die Definition von Resilienz gibt, sondern diese jeweils auf die Zielsetzung einer Studie abgestimmt werden muss, können Befunde mit engen Resilienzkriterien auch nicht einfach generalisiert werden (vgl. Lösel und Bender 2007, S. 61).

Trotz des Fehlens einheitlicher Kriterien wird aber der relationale Charakter von Resilienz deutlich. Damit ist gemeint, dass sich Resilienz erst aus einer bestimmten Beeinträchtigungs-Ressourcen-Konstellation der herangezogenen Kriterien definiert (vgl. Staudinger 1999, S. 343 f.). Der Resilienzbegriff hat damit auch tautologischen Charakter oder wie Leipold und Greve (2009) dazu meinen: „Resilience is defined by it's success: Resilience is, if you don't overcome adverse developmental conditions, it isn't resilience“ (Leipold und Greve 2009, S. 40). Kinder, die sich trotz Risiko gut entwickeln, sind also per Definition resilient. Wird die gute Entwicklung trotz Risikos mit deren Resilienz erklärt, ist ein zirkulärer Schluss gezogen. Für die empirische Erfassung von Resilienz ist das entscheidend, da es bedeutet, dass ein Verständnis von Resilienz als Persönlichkeitseigenschaft und damit einherge- 
hend eine direkte Messung von Resilienz - beispielsweise mittels Fragebogenitems - mit der relationalen Auffassung nicht vereinbar ist (vgl. Staudinger 1999, S. 344). Trotz dieser weitverbreiteten relationalen Auffassung von Resilienz sind vor allem in der Psychologie Ansätze verbreitet, die Resilienz als relativ stabiles Persönlichkeitsmerkmal verstehen (z. B. Block und Block 1980) und versuchen, Resilienz über psychometrische Skalen mittels Fragebögen zu messen (z. B. Wagnild und Young 1993; Schumacher et al. 2005; Leppert et al. 2008). Vertreter dieser Richtung räumen ein, dass sich dabei erhebliche Abgrenzungsprobleme zu anderen verwandten Konzepten (vgl. Schumacher et al. 2005), wie etwa „Kohärenzgefühl“ (Antonovsky 1987), „Hardiness“ (Kobasa 1982) oder „Selbstwirksamkeitserwartung“ (Bandura 1997) ergeben. Gerade Konstrukte wie Selbstwirksamkeitserwartung werden im relationalen Ansatz als Schutzfaktoren angesehen und stellen ein wichtiges Bestimmungsstück von Resilienz dar. Entscheidend ist, in welchem Zusammenhang der Schutzfaktor mit dem Risikofaktor und dem Entwicklungsergebnis steht. Beim Versuch, Resilienz direkt zu messen, muss notwendigerweise eine starke Konfundierung der Konzepte vorliegen. Während psychometrische Resilienzskalen eine allgemeine persönliche Widerstandsfähigkeit unterstellen, definiert sich Resilienz als relationales Konstrukt aus den herangezogenen Kriterien. Würde man als Gedankenexperiment versuchen, eine allgemeine Resilienzskala in ein relationales Modell einzubeziehen, müsste eine solche Skala das Entwicklungsergebnis unter Berücksichtigung des Risikofaktors exakt vorhersagen können, um den Anspruch zu erfüllen, die Resilienz valide gemessen zu haben. Anders ausgedrückt: Sie müsste die verbleibenden Residuen (die Abweichung der beobachteten Werte von den durch das Modell vorhergesagten Werten) bei Konstanthaltung des Risikoeinflusses abbilden. Man könnte also auf die psychometrische Messung verzichten und direkt die Residuen errechnen, um sie als Resilienzmaß anzusehen.

Wenngleich es nicht üblich ist, Resilienz direkt als Residuen abzubilden, kommt das Prinzip häufig implizit zur Anwendung, nämlich dann, wenn danach gefragt wird, ob ein potenzieller Schutzfaktor ein vorhandenes Risiko kompensieren kann. $\mathrm{Zu}$ diesem Zweck wird untersucht, ob der Schutzfaktor unter Berücksichtigung des Risikos einen positiven Effekt auf das Entwicklungsergebnis hat. Ein solcher Effekt wird als Resilienz interpretiert (z.B. Jäkel et al. 2012). Technisch ist dies gleichbedeutend mit der Untersuchung der Korrelation der protektiven Variable mit den verbleibenden Residuen. Je stärker die Korrelation, desto protektiver der Faktor (da mit Resilienz assoziiert). Auch die vielzitierte Kauai-Studie von Emmy Werner und Ruth Smith (1982), der die Fachwelt die wichtigsten und umfangreichsten empirischen Befunde zu protektiven Faktoren zu verdanken hat, ging im Grunde nach diesem Prinzip vor. Hier wurde verglichen, in welchen Merkmalen sich Risikokinder, die zuvor aufgrund ihrer Entwicklung als resilient oder vulnerabel definiert wurden, unterschieden, um diese Merkmale schließlich als protektive Faktoren zu interpretieren. Allerdings ist auch eine solche Auffassung protektiver Faktoren nicht unproblematisch, da aus Sicht der Risikoforschung die Gefahr besteht, lediglich die „Kehrseite der Medaille“ zu betrachtet, also das Fehlen von Risiken und somit lediglich Risikoforschung mit umgekehrten Vorzeichen betrieben wird (vgl. Laucht 1999, S. 306). Eine nähere Betrachtung des Risiko- und Schutzfaktorenkonzeptes ist daher nötig. 
Als Risikofaktor gilt laut Garmezy (1983) ein Merkmal, ,,das bei einer Gruppe von Individuen, auf die dieses Merkmal zutrifft, die Wahrscheinlichkeit des Auftretens einer Störung im Vergleich zu einer unbelasteten Kontrollgruppe erhöht.“ (Laucht 1999, S. 3). Betont wird dabei der probabilistische Charakter von Risikofaktoren. Liegt ein solcher vor, ist die Wahrscheinlichkeit eines nachteiligen Entwicklungsergebnisses erhöht, aber nicht determiniert. Es handelt sich somit um ein Wahrscheinlichkeitskonzept und nicht um ein Kausalkonzept. So weist Fingerle (1999, S. 95) darauf hin, dass selbst die im Sinne ihrer Vorhersehbarkeit „besten“ Risikofaktoren nicht notwendigerweise unerwünschte Entwicklungsresultate nach sich ziehen (vgl. Wustmann 2004, S. 36). Darin liegt ja auch die Bedeutung von Resilienz: Wäre der Schaden durch den Risikofaktor mit Sicherheit vorhersagbar, gäbe es keine resilienten Kinder. In diesem Sinne beschreibt Fingerle (2007, S. 300) Resilienz als die Gegenwahrscheinlichkeit der Wahrscheinlichkeit für einen negativen Entwicklungsverlauf. „Resilienz - und hierin liegt eine der wesentlichen Bedeutungen dieses Konzepts - belegt die probabilistische Natur der Wirkung von Risikofaktoren: sie wirken nicht zwangsläufig, nicht deterministisch, sondern nur mit einer bestimmten Wahrscheinlichkeit“ (Fingerle 2007, S. 300). Im Fokus stehen in der Regel Lebensbedingungen, die die kindliche Entwicklung beeinträchtigen können, sowie Gruppen von Kindern, deren Entwicklung gefährdet ist, sogenannte Risikokinder. In der Risikoforschung werden proximale und distale Risikofaktoren unterschieden. Während proximale Faktoren sich direkt auf das Kind auswirken, wie zum Beispiel Streitigkeiten der Eltern oder ungünstige Erziehungspraktiken, werden die Auswirkungen distaler Faktoren wie chronische Armut, Trennung/Scheidung der Eltern oder elterliche Psychopathologie indirekt über proximale Faktoren vermittelt (vgl. Wustmann 2004, S. 37). Treten beispielsweise ungünstige kindliche Entwicklungen in Trennungs- bzw. Scheidungsbeziehungen häufiger auf, weil diese häufiger von innerfamiliären Konflikten betroffen sind und diese Konflikte unmittelbar beeinträchtigend wirken, so wird der Risikofaktor Trennung/Scheidung als lediglich distal und der Faktor Konflikte als proximal bezeichnet. ${ }^{2}$

Risikomildernde, Schutz- oder protektive Faktoren wurden als positiver Gegenbegriff zu dem der Risikofaktoren konzipiert. Protektive Faktoren sind psychologische Merkmale oder Eigenschaften der sozialen Umwelt, welche die Auftretenswahrscheinlichkeit psychischer Störungen senken bzw. die Auftretenswahrscheinlichkeit eines positiven bzw. gesunden Ergebnisses erhöhen (vgl. Wustmann 2004, S. 44). Uneinigkeit besteht jedoch darüber, wie der Schutzmechanismus zu denken ist. Wie schon angedeutet, kommt es zu terminologischen Verwirrungen, wenn protektive Merkmale im Sinne eines Fehlens von Risiken lediglich als „Kehrseite der Medaille“ verstanden werden. In diesem Fall stellen Risiko- und Schutzfaktoren lediglich gegenüberliegende Pole ein- und desselben Merkmals dar. So hält etwa Laucht (1999, S. 306) kritisch fest, dass viele der ermittelten protektiven Faktoren aus der Liste der Kauai-Studie (Werner und Smith 1982) bereits aus Katalogen von Risikofaktoren bekannt seien - nur eben als Gegenpole oder als das Fehlen dieser Merkmale definiert. Beispielsweise gilt eine emotionale stabile Bindung an eine Bezugsperson als

\footnotetext{
${ }^{2}$ In statistischen Analysen wird bei solchen Zusammenhängen auch von direkten und indirekten Effekten (bzw. Mediation) gesprochen.
} 
Schutzfaktor. Ebenso wird aber dessen Fehlen in der Entwicklungspsychopathologie als ernstes Störrisiko betrachtet (vgl. Wustmann 2004, S. 44). Mehrere AutorInnen haben daher auf die konzeptionellen Schwierigkeiten des Schutzansatzes hingewiesen (z.B. Rutter 1985; Laucht 1999) und ausdrücklich gefordert, risikoerhöhende und risikomildernde Faktoren klar voneinander abzugrenzen und klare Vorstellungen darüber zu formulieren, wie Risiko- und Schutzfaktoren zusammenwirken. Rutter (1990) betont in diesem Zusammenhang, dass nur dann von einem protektiven Faktor gesprochen werden sollte, wenn dieser die schädliche Wirkung des Risikofaktors moderiert, d. h. wenn durch Vorliegen des protektiven Merkmals der Risikoeffekt gemindert oder völlig beseitigt wird. Bei Fehlen des protektiven Merkmals kommt der Risikoeffekt voll zum Tragen. Dahinter steht die Vorstellung einer Pufferwirkung: Der Schutzfaktor verringert die Einflussstärke des Risikofaktors auf das Entwicklungsergebnis. Methodisch betrachtet bedeutet dies den Nachweis eines spezifischen Interaktionseffekts zwischen Risiko- und Schutzfaktor. ${ }^{3}$ Noch strenger ausgelegt wäre ausschließlich dann von einem Schutzfaktor zu sprechen, wenn dieser nur bei Vorliegen einer Gefährdung wirksam wird, ihm ohne Gefährdung aber keine besondere Rolle zukommt (vgl. Laucht 1999, S. 308). Laucht (1999) kritisiert, dass viele ForscherInnen nicht zuletzt wegen der methodischen Schwierigkeiten bei der statistischen Prüfung von Interaktionseffekten offensichtlich bereit sind, eine viel einfachere Vorstellung über das Zusammenspiel von Risiko- und Schutzfaktoren zu akzeptieren, bei der lediglich erwartet wird, dass von einem protektiven Faktor ein gesundheitsförderlicher Einfluss ausgeht. Dieser wird dann aber nicht nur auf die Risikogruppe beschränkt, sondern hat den Charakter eines allgemein förderlichen Effekts, der für jedes Individuum gleichermaßen gilt. ${ }^{4}$ Hier wird das Problem der Annahme einer protektiven Wirkung deutlich. Denn wogegen werden Kinder der Nichtrisikogruppe geschützt? Der Nachweis eines Interaktionseffekts hingegen, bei dem der Schutzfaktor (als Moderatorvariable) den Einfluss des Risikofaktors egalisiert oder zumindest abschwächt, würde die Annahme eines protektiven Effekts rechtfertigen.

\section{Resilienzmodelle}

Für die empirische Erfassung von Resilienz erscheinen aus den bisherigen Ausführungen folgende Überlegungen wesentlich, die in weiterer Folge zu bestimmten Resilienzmodellen führen: Um von Resilienz sprechen zu können, muss zunächst ein wünschenswertes Ergebnis (Outcome) definiert werden und eine signifikante Bedrohung (Risiko) zur Erreichung des Ergebnisses feststellbar sein. Risiko und Ergebnis müssen einen Zusammenhang aufweisen, d.h. das Risiko stellt einen Prädiktor für

\footnotetext{
${ }^{3}$ Liegt so ein Interaktionseffekt vor, fungiert der Schutzfaktor als Moderatorvariable: Sie moderiert den Zusammenhang zwischen dem Prädiktor (hier der Risikofaktor) und dem Outcome, d.h. je nach Ausprägung der Moderatorvariable ändert sich die Zusammenhangsstärke zwischen Prädiktor und Outcome.

${ }^{4}$ Mathematisch betrachtet handelt es sich um eine additive Verknüpfung der Einflüsse von Risiko- und Schutzfaktoren, wobei Risiken und protektive Faktoren einfach mit umgekehrten Vorzeichen eingehen (Laucht 1999, S. 309).
} 
ein unerwünschtes Ergebnis dar. Bei geringem Risiko wird ein besseres Ergebnis erwartet, bei hohem Risiko ein schlechteres. Ersteres (geringes Risiko - gutes Outcome) bedeutet die Norm des Wohlergehens, Letzteres (hohes Risiko - schlechtes Outcome) steht für Vulnerabilität, die in diesem Sinne ebenfalls die Norm darstellt. Resilienz hingegen drückt sich in der Abweichung von der Norm aus (hohes Risiko - gutes Outcome). Denkbar ist noch eine weitere abweichende Konstellation (geringes Risiko - schlechtes Outcome), die auf hochgradig vulnerable Kinder hinweisen würde. Wird solch eine Typologie aufgrund kategorialer Variablen erstellt, handelt es sich nach Masten (2001) um einen personen-fokussierten Ansatz der Resilienzforschung. Das klassische Design, wie es beispielsweise die Kauai-Studie von Werner und Smith (1982) darstellt, identifiziert unter Risikokindern vulnerable und resiliente und vergleicht sie hinsichtlich möglicher Schutzfaktoren. In erweiterten Designs können auch Nicht-Risikokinder berücksichtigt werden, um zu vergleichen, wie sich resiliente Kinder von Kindern, die ohne Risiko im Wohlergehen aufwuchsen, unterscheiden. Die vierte Gruppe (geringes Risiko - schlechtes Outcome) stellte laut Masten (2001, S. 233) in empirischen Studien meist ein „EmptyCell"“-Phänomen dar, d.h. in Untersuchungen war die Zahl der zu beobachtenden Fälle zu gering, um Berücksichtigung zu finden (z.B. Luthar und Cushing 1999; Masten et al. 1990). Während personen-fokussierte Ansätze darauf abzielen, Merkmalsprofile von Gruppen von Kindern zu vergleichen, um auf protektive Faktoren zu schließen, stellen variablen-fokussierte Ansätze (vgl. Masten 2001) statistische Modelle dar, die das spezifische Zusammenspiel zwischen Risiko-, Schutzfaktoren und Entwicklungsergebnis beschreiben. Wie schon gezeigt, sind in multivariaten Modellen verschiedene Konstellationen des Zusammenwirkens denkbar. Masten (2001) hat eine Systematisierung verschiedener Resilienzmodelle vorgelegt, in der sie zwischen a) Haupteffekt-Modellen, b) Mediations-Modellen und c) Interaktionsmodellen unterscheidet. Haupteffekte drücken den direkten Einfluss von risikoerhöhenden oder risikovermindernden Bedingungen auf das Entwicklungsergebnis aus, wenngleich kausale Zusammenhänge in solchen Korrelationsanalysen nicht nachweisbar sind. Ein Haupteffektmodell beschreibt die additive Verknüpfung von Einflussfaktoren, die - wie bereits diskutiert - je nach Richtung der Betrachtungsweise als risikomindernd oder risikoerhöhend interpretiert werden können. Im Mediationsmodell wirken risikoerhöhende und risikomildernde Faktoren indirekt über eine Mediatorvariable auf das Entwicklungsergebnis. Beispielsweise wird die kindliche Entwicklung direkt vom Erziehungsverhalten der Eltern beeinflusst, das Erziehungsverhalten selbst hingegen unterliegt dem Einfluss weiterer Risiko- oder Schutzfaktoren. Im Interaktionsmodell fungiert der potenzielle Schutzfaktor als Moderatorvariable, die die Einflussstärke des Risikofaktors auf das Entwicklungsergebnis abschwächt, je stärker sie ausgeprägt ist.

\section{Ziele der vorliegenden Untersuchung}

In den folgenden empirischen Analysen zu kindlichem Wohlbefinden soll in Anlehnung an die Systematisierung von Masten (2001) die analytische Trennung zwischen additiven Effekten, Mediatoreffekten und Moderatoreffekten vorgenommen werden. 
Dazu einige methodische Vorüberlegungen: Weist ein potenzieller Schutzfaktor nur einen additiven Effekt auf, bedeutet es, dass er einen positiven Beitrag zum Outcome leisten kann - allerdings unabhängig vom Risikofaktor. ${ }^{5}$ Interventionsmaßnahmen, die auf diesem Modelltyp beruhen, zielen in der Regel darauf ab, risikomildernde Faktoren zu erhöhen. Dahinter steht die Idee, dass bei genügend risikomildernden Faktoren das Entwicklungsergebnis derart beeinflusst wird, dass der Effekt der Risikofaktoren kompensiert wird. Inwieweit hier bereits von Resilienz bzw. von einem protektiven Effekt gesprochen werden kann, ist eine in der Fachwelt nicht geklärte analytische/definitorische Frage. Bei einem Mediatoreffekt wirken Einflussfaktoren indirekt über eine Mediatorvariable auf das Entwicklungsergebnis. Die Mediatorvariable zeigt direkten Einfluss auf das Entwicklungsergebnis, steht aber auch mit den Einflussfaktoren in Zusammenhang. Wird die Mediatorvariable als Schutzfaktor gesehen, ist der Zusammenhang meist derart, dass mit geringerem Risiko auch eine höhere Ausprägung der protektiven Variable assoziiert ist. Damit liegt eine Konfundierung zwischen Risiko- und Schutzfaktor vor. Auch bei dieser Konstellation ist die protektive Wirkung fraglich. Mehr noch: Während ein additiver Effekt den Zusammenhang des Schutzfaktors mit der durch den Risikofaktor nicht erklärten Varianz (Residuen) der Outcomevariable ausdrückt und somit die Abweichung von der Norm (=Resilienz) erklärt, steht der Mediationseffekt eben für jenen Varianzanteil der Outcomevariable, den Schutzfaktor und Risikofaktor gemeinsam erklären - also eben jenen Varianzanteil, der nicht für Resilienz steht. Somit liegt eigentlich kein Schutzeffekt vor. Der Schutzfaktor ist eher umgekehrt betrachtet als proximaler Risikofaktor (z. B. mangelhafte Erziehungsqualität) zu sehen, der die Wirkung eines distalen Risikofaktors (z. B. Armut, Trennung/Scheidung der Eltern) vermittelt. Besteht beispielsweise in einer armen Familie ein harmonisches Klima und günstige Erziehungsqualität, verliert der distale Faktor seine Risikofunktion. Auch Lösel und Bender (2007, S. 63) weisen darauf hin, dass betreffende Kinder eigentlich nicht resilient sind, sondern nur einem geringeren Risiko ausgesetzt gewesen sind, als jene, bei denen distale und proximale Risiken zusammenkommen. Bei einem Moderatoreffekt schließlich schwächt der Schutzfaktor den Effekt des Risikofaktors ab: Je höher die Ausprägung des Schutzfaktors, desto geringer wird der Zusammenhang zwischen Risikofaktor und Outcomevariable. Resilienz kann hier als Schutzfunktion der Moderatorvariable im Sinne eines abpuffernden Effekts gesehen werden. Ist der Schutzfaktor stärker, wird der Einfluss des Risikos schwächer.

Um dem Resilienzphänomen näher auf den Grund zu gehen, gilt es also zu untersuchen, welche Art von Effekt in den einzelnen Kontexten tatsächlich vorliegt. Mediation und Moderation schließen sich nicht aus. In aller Regel wird ein potenzieller Schutzfaktor in mehrerlei Hinsicht zum Outcome beitragen. Meist wird ein Schutzfaktor einen eigenständigen Erklärungsbeitrag leisten (additiver Effekt), einen Teil der erklärbaren Varianz wird er aber mit dem Risikofaktor teilen (Mediatoreffekt). Kommt ein Moderatoreffekt hinzu, wird dieser selten in reiner Form auftreten, sondern eher in Kombination mit einem additiven Effekt und Mediatoreffekten. Um

\footnotetext{
5 Statistisch gesprochen liegt ein additiver Effekt vor, wenn Risikofaktor und Schutzfaktor untereinander keine Korrelation aufweisen und somit bei der Erklärung des Outcomes in einem multivariaten Modell keine Konfundierung vorliegt.
} 
Moderatoreffekte zu untersuchen, betrachtet man, ob die potenzielle Moderatorvariable Einfluss auf die Zusammenhangsstärke zwischen Prädiktor und Kriterium hat. Je nach Datenqualität (Skalenniveau) kann dabei verschieden vorgegangen werden. Sind alle Variablen metrisch skaliert und lineare Zusammenhänge zu unterstellen, wird der Einbezug von Interaktionstermen (Prädiktor mal Moderator) in ein Regressionsmodell vorgeschlagen (vgl. Aiken und West 1991). Im Falle von kategorialen Variablen bietet sich die mehrdimensionale Tabellenanalyse an (Kühnel und Krebs 2001).

Im Folgenden soll anhand der Daten einer 2013 durchgeführten Kinderstudie untersucht werden, welche Auswirkungen sozioökonomische Risiken auf kindliches Wohlbefinden haben und wie vorhandene Ressourcen als potenzielle Schutzfaktoren diese Auswirkungen beeinflussen. Von Interesse ist dabei, inwieweit in verschiedenen Lebensbereichen additive Effekte, Mediatoreffekte und Moderatoreffekte potenzieller Risiko- und Schutzfaktoren vorliegen, um zu sehen, ob und inwieweit in diesen Bereichen von Resilienzphänomenen gesprochen werden kann.

\section{Methoden}

\subsection{Stichprobe}

Die Daten stammen aus einer Studie, die objektive Lebensbedingungen und subjektive Lebensqualität von Kindern im Unterstufenalter untersuchte. Dazu wurden im Jahr 2013 schriftliche Befragungen in neun Schulen im österreichischen Bundesland Steiermark durchgeführt. Die Schulen sind in der Landeshauptstadt Graz sowie im umliegenden ländlichen Raum angesiedelt und umfassten die Schultypen Hauptschule, Neue Mittelschule sowie Allgemeinbildende Höhere Schule (Unterstufe). Die ursprüngliche Gesamtstichprobe umfasste 1756 Kinder und Jugendliche aller Schulstufen der Sekundarstufe 2 im Alter zwischen 10 und 15 Jahren, von denen $48 \%$ weiblich und $52 \%$ männlich waren. Die Stichprobe ist das Ergebnis einer bewussten Auswahl an Schulen mit dem Ziel, möglichst hohe Varianz hinsichtlich der Merkmale Stadt/Land und Schultyp zu erreichen. Es handelt sich somit um keine repräsentative Stichprobe im Sinne einer Zufallsauswahl. Da das Ziel der ursprünglichen und vorliegenden Analysen auch nicht bei der repräsentativen Schätzung von Populationsparametern, sondern in der Untersuchung von Merkmalszusammenhängen lag, wurde auf die Ziehung einer steiermarkweiten Zufallsstichprobe zugunsten einer theoretisch begründeten und für differenzierte Auswertungen hinreichend groBen Stichprobe verzichtet. Die realisierte Stichprobe verteilt sich sowohl auf die Schultypen (47,7\% NMS/HS und 52,3\% AHS-Unterstufe) als auch auf die Region (48,1\% Stadt und 51,9\% Land) in etwa gleich. Die verbleibenden Abweichungen von der Gleichverteilung hinsichtlich der Merkmale Region und Schultyp wurden durch entsprechende Gewichtungsfaktoren ausgeglichen. Aufgrund einiger fehlender Angaben in der Variable Wohnort beinhaltet der gewichtete Datensatz, der die Basis der folgenden Analysen darstellt, eine etwas geringere Fallzahl von $N=1749$. Die ursprüngliche Studie verstand sich als Beitrag zu den ,neuen“ Ansätzen der sozialwissenschaftlichen Kindheitsforschung, die im Gegensatz zu traditionellen so- 
zialisationstheoretischen und entwicklungspsychologischen Ansätzen darauf zielt, die Perspektive der Kinder einzunehmen und Lebensbereiche nicht im Hinblick auf ihre Sozialisationsfunktionen zu bewerten, sondern dahingehend zu analysieren, welchen Beitrag sie zum Wohlbefinden der Kinder leisten und welche Chancen zur Teilhabe sie Kindern einräumen (vgl. Wilk und Bacher 1994). Folglich soll Wohlbefinden nicht an von der Erwachsenenwelt vorgegebenen Normen guter Entwicklung bestimmt werden, vielmehr geht es um das aktuelle Wohlbefinden der Kinder als Subjekte im Hier und Jetzt (siehe auch Zander 2008, S. 16). Forschungsmethodisch bedeutet dies, sich auf die Selbstaussagen der Kinder zu stützen und die Kinder selbst als die Expertinnen und Experten ihres Lebens ernst zu nehmen (vgl. Bock 2010; Hurrelmann und Andresen 2010). ${ }^{6}$

\subsection{Variablen}

Für das subjektive Wohlbefinden der Kinder als Outcome-Variable wurde als Indikator die globale Frage nach der allgemeinen Lebenszufriedenheit herangezogen (,Wie zufrieden bist du mit deinem Leben insgesamt?“), die über eine fünfstufige Gesichter-Skala beantwortet werden konnte $(1=\operatorname{sehr}$ zufrieden, $5=$ gar nicht zufrieden $).^{7}$

Als sozioökonomische Risiken wurden Trennung/Scheidung der Eltern sowie das Aufwachsen in Armutslagen untersucht. Während nach einer Trennung/Scheidung der Eltern direkt gefragt werden kann, ist es bei Kinderstudien, in denen Eltern nicht mitbefragt wurden, schwieriger, den sozioökonomischen Herkunftsstatus zu bestimmen. Für die Analyse standen zum einen die Familienwohlstandsskala ${ }^{8}$, zum anderen ein Index zu subjektiven Armutserfahrungen zur Verfügung. Erste Analysen zeigten, dass die an objektiveren Kriterien gemessene Familienwohlstandsskala im Gegensatz zu den subjektiven Armutserfahrungen der Kinder keinen direkten Einfluss auf deren Lebenszufriedenheit zeigte. Dies deutet darauf hin, dass ökonomische Einschränkungen nicht an sich, jedoch deren subjektives Erleben bedeutsam für das Wohlbefinden von Kindern sind. Die weiteren Analysen wurden daher mit dem Index für subjektive Armutserfahrungen vorgenommen. Dieser wurde nach dem Vorbild der World Vision Kinderstudie (vgl. Hurrelmann und Andresen 2010) konstruiert. Kinder wurden gebeten, die Aussagen „Das Geld ist bei uns öfter knapp“

\footnotetext{
${ }^{6}$ Erwachsene als InformantInnen über Kinder zu befragen, erscheint bei Fragen nach objektiven Gegebenheiten (z. B. berufliche Situation der Eltern) sinnvoll, die für Kinder schwer zu beantworten sind. Neben der Frage nach einer Trennung/Scheidung der Eltern, die von Kindern und Jugendlichen dieser Altersgruppe leicht beantwortet werden kann, wurden hier nur Fragen nach subjektiven Einschätzungen analysiert.

7 Auf die Hinzunahme von spezifischen Indikatoren der Lebenszufriedenheit wie etwa der Zufriedenheit mit der Zuwendung der Eltern oder der Zufriedenheit mit der finanziellen Lage der Familie wurde bewusst zugunsten des globalen Indikators verzichtet, um zu vermeiden, dass Prädiktor und Kriterium aufgrund ihrer Ähnlichkeit die gleiche Dimension abbilden und somit zwar hohe, aber inhaltlich triviale Korrelationen erzeugen.

8 Da Kinder nicht einfach nach dem Haushaltsnettoeinkommen ihrer Familie gefragt werden können, wird versucht, Wohlstandsindikatoren zu erfassen, die Kinder leicht beantworten können. Als Wohlstandsindikatoren wurden neben der Frage nach dem eigenen Zimmer auch die Anzahl der Autos in Familienbesitz, Anzahl der Urlaube in den vergangenen 12 Monaten und Anzahl der Computer in der Familie erfragt. Die Kombination der Fragen führt zu einer 10-stufigen Wohlstandsskala (vgl. Dür und Griebler 2007, S. 64).
} 
und „Wir können uns alles leisten, was wir wollen“ mit den Antwortmöglichkeiten „,eher ja“ oder ,eher nein“ zu beurteilen. Die beiden Aussagen wurden zu einem dichotomen Index kombiniert ( $1=$ keine Armutserfahrung, $2=$ Armutserfahrung in mindestens einer Aussage).

Als potenzielle Schutz-/Resilienzfaktoren wurden die Konstrukte elterliche $\mathrm{Zu}$ wendung, Wertschätzung der eigenen Meinung sowie Selbstwirksamkeitserwartung untersucht. Die Operationalisierung orientierte sich ebenfalls an der World Vision Kinderstudie (vgl. Hurrelmann und Andresen 2010). Kinder wurden nach ihrem Empfinden gefragt, ob jeweils ihre Mutter und ihr Vater ausreichend Zeit für sie hätten, was sie mit ,ja“, ,nein“, „,mal so mal so“ und ,ich habe keine Mutter“ bzw. „ich habe keinen Vater" beantworten konnten. Die beiden Fragen wurden zu einem 6-stufigen Index kombiniert, bei dem höhere Werte für geringere wahrgenommene Zuwendung stehen $(1=$ von beiden Elternteilen hinreichend, $6=$ von beiden Elternteilen nicht hinreichend). Weiters wurden die Kinder gefragt, welche Personen eher viel und welche eher wenig Wert auf ihre Meinung legen würden. Dies konnte jeweils für die Personen Mutter, Vater, Freundinnen/Freunde sowie Klassenlehrer/in mit ,eher viel“, „teils/teils“, „,eher wenig“ und „habe ich nicht“ bewertet werden. Die ersten drei Kategorien der vier Variablen wurden zu einem Summenindex zusammengefasst, bei dem höhere Werte gesamt ein geringeres Ausmaß an Wertschätzung darstellen. Das Merkmal Selbstwirksamkeitserwartung wurde schließlich über neun Items einer 5-stufigen likertskalierten Itembatterie gemessen (Beispielitem: „Schwierige Sachen kriege ich gut hin, wenn ich mich anstrenge.“ $1=$ stimmt genau, $5=$ stimmt gar nicht, Chronbachs $\alpha=0,808$ ). Höhere Werte stehen dabei für ein geringeres Ausmaß an Selbstwirksamkeitserwartung.

\subsection{Analysestrategie}

Mittels hierarchischer Regressionsanalysen wurden für die verschiedenen Kombinationen von sozioökonomischem Risiko, Wohlbefinden und potenziellem Schutzfaktor jeweils drei Modelle gerechnet. Das jeweils erste Modell zeigt den unkontrollierten Effekt des Risikofaktors auf das kindliche Wohlbefinden. Das zweite Modell beinhaltet den potenziellen Schutzfaktor als zusätzlichen Prädiktor. Im dritten Modell wird ein etwaiger Moderatoreffekt geprüft, indem als zusätzlicher Prädiktor der Produktterm zwischen Risiko- und Schutzvariable ins Modell aufgenommen wird. Dadurch lässt sich bestimmen, ob der potenzielle Schutzfaktor additiven, indirekt vermittelnden (Mediation) oder moderierenden Einfluss ausübt, und somit welches Resilienzmodell bei der jeweiligen Konstellation empirisch zutreffend ist. Anders als bei vielen multivariaten Regressionsmodellen sind bei dieser Art der Drittvariablenkontrolle nicht nur die direkten Effekte der Prädiktoren auf das Kriterium von Interesse, sondern vor allem die Veränderungen, die sich zeigen, wenn die zusätzlichen Prädiktoren in das Modell aufgenommen werden. ${ }^{9}$ Bei nachweisba-

\footnotetext{
${ }^{9}$ Bei der Konstruktion von Produkttermen wird in der Methodenliteratur die vorherige Zentrierung der Variablen zum Zweck der Vermeidung von Multikollinearität sowie zur erleichterten Interpretierbarkeit empfohlen (vgl. Aiken und West 1991). Die hier verwendeten Prädiktoren wurden zuvor mittels z-Transformation standardisiert, die eine Zentrierung beinhalten.
} 
ren Moderatoreffekten werden die spezifischen Zusammenhänge zusätzlich durch mehrdimensionale Kreuztabellen illustriert.

\section{Ergebnisse}

\subsection{Deskriptive Statistiken}

Abb. 1 gibt die Häufigkeitsverteilung der zu erklärenden Variable Allgemeine Lebenszufriedenheit wieder. Knapp zwei Drittel der befragten Kinder und Jugendlichen $(66,4 \%)$ gaben an, mit ihrem Leben sehr zufrieden zu sein. Ein weiteres Viertel $(25,6 \%)$ war mit dem Leben eher zufrieden. Die restlichen Kinder verteilen sich auf die übrigen Kategorien von teils/teils bis gar nicht zufrieden. Der Mittelwert auf der 5-stufigen Skala lag bei 1,4 mit einer Standardabweichung von 0,7.

Knapp ein Viertel der befragten Kinder und Jugendlichen (23,3\%) gab an, dass ihre Eltern getrennt oder geschieden waren. Der Anteil an Kindern und Jugendlichen mit Armutserfahrungen lag bei 12,5\%. Abb. 2 gibt die Häufigkeitsverteilung des Zuwendungsindex wieder. Mehr als die Hälfte der Kinder $(53,1 \%)$ berichten über ausreichend Zuwendung von beiden Elternteilen. Der Mittelwert im 6-stufigen Zuwendungsindex lag bei 1,24 mit einer Standardabweichung von 1,20.

Abb. 1 Allgemeine Lebenszufriedenheit

Abb. 2 Index Zuwendung
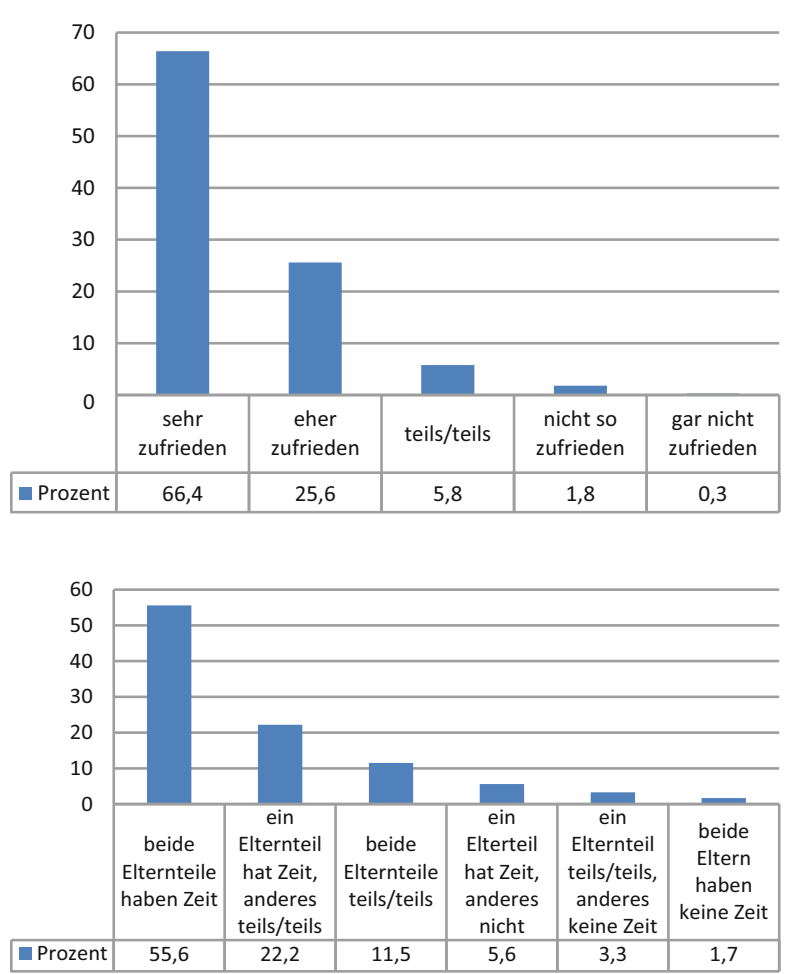
Der Mittelwert im Index zur Wertschätzung der eigenen Meinung $(1=$ hohe Wertschätzung bis 3=geringe Wertschätzung) beträgt 1,6 mit einer Standardabweichung von 0,4. Der Mittelwert in der Skala Selbstwirksamkeitserwartung, welcher als Summenindex die Werte 9 (=hohe Selbstwirksamkeit) bis 36 (=geringe Selbstwirksamkeit) annehmen konnte, lag bei 14,9 mit einer Standardabweichung von 4,0.

\subsection{Trennung/Scheidung der Eltern und Lebenszufriedenheit}

\subsubsection{Elterliche Zuwendung als Schutzfaktor}

Tab. 1 gibt die Ergebnisse der hierarchischen Regressionsanalyse wieder, in der der Effekt einer Trennung/Scheidung der Eltern auf die allgemeine Lebenszufriedenheit der Kinder unter Berücksichtigung der elterlichen Zuwendung untersucht wurde.

Modell 1 zeigt zunächst einen signifikanten bivariaten Effekt $\left(\beta=-0,119^{* *}\right)$, der darauf hinweist, dass sich eine Trennung/Scheidung der Eltern ungünstig auf die Lebenszufriedenheit auswirkt. Bei Berücksichtigung der wahrgenommenen elterlichen Zuwendung (Modell 2) zeigt sich aber, dass der Effekt der Trennung/Scheidung verschwindet $(\beta=-0,038$ n.s.), sich also der ursprüngliche bivariate Effekt vollständig über die wahrgenommene Zuwendung erklären lässt. Je mehr Zuwendung wahrgenommen wird, desto höher ist die Lebenszufriedenheit $\left(\beta=0,375^{* *}\right)$. Modell 2 zeigt eine signifikante Steigerung in der erklärten Varianz der abhängigen Variable von $\mathrm{R}^{2}=0,014 * *$ auf $\mathrm{R}^{2}=0,148^{* *}$. Die zusätzliche Berücksichtigung der Interaktion (Modell 3) zwischen Trennung/Scheidung der Eltern und Zuwendung bringt hingegen keine signifikante Verbesserung der Varianzaufklärung. Die wahrgenommene Zuwendung bleibt in diesem Modell der einzige signifikante Prädiktor $\left(\beta=0,385^{* *}\right)$. Zuwendung fungiert in diesem Modell also als Mediatorvariable zwischen Trennung/Scheidung der Eltern und Lebenszufriedenheit. Dies lässt darauf schließen, dass eine Trennung/Scheidung der Eltern sich per se nicht negativ auf die Lebenszufriedenheit auswirkt, jedoch das Risiko, weniger Zuwendung zu erfahren, bei Vorliegen einer Trennung/Scheidung vergleichsweise erhöht ist. Ein Mangel an Zuwendung wirkt sich in direkter Weise ungünstig auf die Lebenszufriedenheit aus. Zuwendung zeigt also weder im kompensatorischen Sinn (additiver Effekt) noch im Sinne eines Abpuffereffekts (Moderation) eine Schutzfunktion. Eher kann ein Mangel an Zuwendung als ein direkter (proximaler) Risikofaktor gesehen werden,

Tab. 1 Hierarchische Regressionsanalyse. Abhängige Variable: Allgemeine Lebenszufriedenheit ( 1 = sehr zufrieden, 5 = gar nicht zufrieden)

\begin{tabular}{llll}
\hline & Modell 1 & Modell 2 & Modell 3 \\
& $\beta$ & $\beta$ & $\beta$ \\
\hline Eltern getrennt/geschieden (1= ja, 2= nein) & $-0,119^{* *}$ & $-0,038$ & $-0,046$ \\
Zuwendung (1= hoch bis 6= niedrig) & - & $0,375^{* *}$ & $0,385^{* *}$ \\
Interaktion Eltern getrennt/geschieden*Zuwendung & - & - & 0,046 \\
$R^{2}$ & $0,014 * *$ & $0,148^{* *}$ & $0,150^{* *}$ \\
Änderung in $R^{2}$ & - & $0,134 * *$ & 0,002 \\
\hline
\end{tabular}

Anmerkung: $* * p<0,010$ 
der den Einfluss des indirekten (distalen) Risikofaktors (Trennung/Scheidung der Eltern) vermittelt.

\subsubsection{Wertschätzung der eigenen Meinung als Schutzfaktor}

In Tab. 2 wurde anstelle der wahrgenommenen Zuwendung die wahrgenommene Wertschätzung der eigenen Meinung als möglicher Schutzfaktor untersucht.

Der Vergleich von Modell 2 mit Modell 1 zeigt, dass die Wertschätzung zusätzliche Erklärungsstärke bringt, ohne dass der ursprüngliche bivariate Effekt verschwindet. Wertschätzung wirkt sich also positiv auf die Lebenszufriedenheit aus, egalisiert aber nicht den Effekt einer Trennung/Scheidung der Eltern. Die zusätzliche Berücksichtigung der Interaktion zwischen Trennung/Scheidung der Eltern (Modell 3) und der Wertschätzung zeigt einen schwachen, aber signifikanten Moderatoreffekt. Dies weist darauf hin, dass die Stärke des Einflusses einer Trennung/Scheidung auf die Lebenszufriedenheit von der jeweiligen Ausprägung der Wertschätzung abhängig ist. Um dies zu veranschaulichen, wurden die Zusammenhänge der drei Variablen in einer trivariaten Kreuztabelle dargestellt. Zur Vereinfachung der Darstellung wurden die Variablen Wertschätzung und Lebenszufriedenheit jeweils an ihrem Mittelwert dichotomiert.

Tab. 2 Hierarchische Regressionsanalyse. Abhängige Variable: Allgemeine Lebenszufriedenheit (1= sehr zufrieden, 5 = gar nicht zufrieden)

\begin{tabular}{llll}
\hline & Modell 1 & Modell 2 & Modell 3 \\
& $\beta$ & $\beta$ & $\beta$ \\
\hline Eltern getrennt/geschieden (1= ja, 2= nein) & $-0,125^{* *}$ & $-0,098^{* *}$ & $-0,094^{* *}$ \\
Wertschätzung (1 = hoch bis 3= niedrig) & - & $0,381^{* *}$ & $0,383^{* *}$ \\
Interaktion Eltern getrennt/gesch.*Wertschätzung & - & - & $-0,049^{*}$ \\
$R^{2}$ & $0,016^{* *}$ & $0,160^{* *}$ & $0,163 * *$ \\
Änderung in $R^{2}$ & - & $0,145^{* *}$ & $0,002^{*}$ \\
\hline
\end{tabular}

Anmerkung: $* p<0,050 ; * * p<0,010$

Tab. 3 Trivariate Kreuztabelle: Abhängige Variable: Allgemeine Lebenszufriedenheit (dichotomiert). Unabhängige Variable: Eltern getrennt/geschieden. Kontrollvariable: Wertschätzung der eigenen Meinung (dichotomiert)

\begin{tabular}{|c|c|c|c|c|c|c|}
\hline \multicolumn{3}{|c|}{ Wertschätzung } & \multicolumn{2}{|c|}{ Eltern getrennt/geschiedenn } & \multirow{2}{*}{$\begin{array}{l}\text { Gesamt } \\
(\%)\end{array}$} & \multirow[b]{2}{*}{ Phi } \\
\hline & & & $\mathrm{Ja}(\%)$ & Nein $(\%)$ & & \\
\hline \multirow[t]{3}{*}{ Hoch } & Zufriedenheit & Hoch & 75,8 & 79,3 & 78,6 & - \\
\hline & & Niedrig & 24,2 & 20,7 & 21,4 & - \\
\hline & Gesamt & & 100,0 & 100,0 & 100,0 & $-0,034$ \\
\hline \multirow[t]{3}{*}{ Gering } & Zufriedenheit & Hoch & 34,7 & 52,1 & 47,7 & - \\
\hline & & Niedrig & 65,3 & 47,9 & 52,3 & - \\
\hline & Gesamt & & 100,0 & 100,0 & 100,0 & $-0,151 * *$ \\
\hline \multirow[t]{3}{*}{ Gesamt } & Zufriedenheit & Hoch & 58,3 & 69,6 & 67,1 & - \\
\hline & & Niedrig & 41,7 & 30,4 & 32,9 & - \\
\hline & Gesamt & & 100,0 & 100,0 & 100,0 & $-0,100 * *$ \\
\hline
\end{tabular}

Anmerkung: $* * p<0,010$ 
Aus Tab. 3 wird ersichtlich, dass der Zusammenhang zwischen Trennung/ Scheidung der Eltern und Lebenszufriedenheit bei hoher Wertschätzung geringer ausfällt als bei geringer Wertschätzung. Bei hoher Wertschätzung zeigt sich, dass 75,8\% der Kinder mit getrennten/geschiedenen Eltern mit ihrem Leben hochzufrieden sind, während es bei Kindern mit zusammenlebenden Eltern 79,3\% sind, also nur 3,5 Prozentpunkte mehr $(\varphi=-0,034$ n.s.). Bei geringer Wertschätzung hingegen sind lediglich $34,7 \%$ der Kinder mit getrennten/geschiedenen Eltern mit ihrem Leben hochzufrieden, während es bei Kindern mit zusammenlebenden Eltern $52,1 \%$ sind. Die Prozentsatzdifferenz beträgt bei geringer Wertschätzung damit $17,4 \%(\varphi=-0,151 * *)$. Somit liegt nur bei geringer Wertschätzung, nicht jedoch bei hoher Wertschätzung, ein signifikanter Einfluss der Trennung/Scheidung der Eltern auf die Lebenszufriedenheit vor. Wertschätzung zeigt in diesem Zusammenhang also nicht nur eine Schutzfunktion im kompensatorischen Sinn (additiver Effekt), sondern tendiert auch zu einem Abpuffereffekt (Moderation) in dem Sinn, dass bei vorliegender Wertschätzung der Trennungs-/Scheidungseffekt seine Bedeutung verliert.

\subsubsection{Selbstwirksamkeitserwartung als Schutzfaktor}

Ein vergleichbares Bild wie bei der wahrgenommenen Wertschätzung zeigt sich auch bei der Berücksichtigung von Selbstwirksamkeitserwartung. Die Ergebnisse sind in Tab. 4 wiedergegeben.

Selbstwirksamkeit erweist sich selbst als starker Prädiktor für die allgemeine Lebenszufriedenheit der Kinder. Es liegt jedoch keine Konfundierung mit Trennung/ Scheidung der Eltern vor. Beide Variablen tragen unabhängig voneinander zur Erklärung der Lebenszufriedenheit bei. Die Berücksichtigung der Interaktion der beiden Variablen zeigt zusätzlich einen signifikanten Moderatoreffekt. Wie sich dieser äußert, ist in Tab. 5 dargestellt.

Die unterschiedlich starken Prozentsatzdifferenzen in den beiden Partialtabellen machen deutlich, dass bei hoher Selbstwirksamkeit der Einfluss einer Trennung/ Scheidung auf die Lebenszufriedenheit geringer ausfällt als bei niedriger Selbstwirksamkeit. Selbstwirksamkeit wirkt sich also nicht nur an sich günstig auf die Lebenszufriedenheit aus, sondern setzt auch in dem Sinne Resilienz frei, dass bei selbstwirksamen Kindern der Trennungs-/Scheidungseffekt seinen Einfluss verliert.

Tab. 4 Hierarchische Regressionsanalyse. Abhängige Variable: Allgemeine Lebenszufriedenheit ( $1=$ sehr zufrieden, $5=$ gar nicht zufrieden)

\begin{tabular}{llll}
\hline & Modell 1 & Modell 2 & Modell 3 \\
& $\beta$ & $\beta$ & $\beta$ \\
\hline Eltern getrennt/geschieden $(1=\mathrm{ja}, 2=$ nein) & $-0,118^{* *}$ & $-0,108^{* *}$ & $-0,105^{* *}$ \\
Selbstwirksamkeit $(9=$ hoch bis $36=$ niedrig) & - & $0,462^{* *}$ & $0,457^{* *}$ \\
Interaktion Eltern getrennt/gesch.*Selbstwirksamk & - & - & $-0,082^{* *}$ \\
$R^{2}$ & $0,014 * *$ & $0,227^{* *}$ & $0,234^{* *}$ \\
Änderung in $R^{2}$ & - & $0,213^{* *}$ & $0,007^{* *}$ \\
\hline
\end{tabular}

Anmerkung: $* * p<0,010$ 
Tab. 5 Trivariate Kreuztabelle: Abhängige Variable: Allgemeine Lebenszufriedenheit (dichotomiert). Unabhängige Variable: Eltern getrennt/geschieden. Kontrollvariable: Selbstwirksamkeitserwartung (dichotomiert)

\begin{tabular}{|c|c|c|c|c|c|c|}
\hline \multicolumn{3}{|c|}{ Selbstwirksamkeit } & \multicolumn{2}{|c|}{ Eltern getrennt/geschieden } & \multirow{2}{*}{$\begin{array}{l}\text { Gesamt } \\
(\%)\end{array}$} & \multirow[b]{2}{*}{ Phi } \\
\hline & & & $\mathrm{Ja}(\%)$ & Nein $(\%)$ & & \\
\hline \multirow[t]{3}{*}{$\overline{\text { Hoch }}$} & Zufriedenheit & Hoch & 77,9 & 83,0 & 81,8 & - \\
\hline & & Niedrig & 22,1 & 17,0 & 18,2 & - \\
\hline & Gesamt & & 100,0 & 100,0 & 100,0 & $-0,056$ \\
\hline \multirow[t]{3}{*}{ Niedrig } & Zufriedenheit & Hoch & 38,6 & 54,2 & 50,5 & - \\
\hline & & Niedrig & 61,4 & 45,8 & 49,5 & - \\
\hline & Gesamt & & 100,0 & 100,0 & 100,0 & $-0,133 * *$ \\
\hline \multirow[t]{3}{*}{ Gesamt } & Zufriedenheit & Hoch & 58,1 & 68,9 & 66,4 & - \\
\hline & & Niedrig & 41,9 & 31,1 & 33,6 & - \\
\hline & Gesamt & & 100,0 & 100,0 & 100,0 & $-0,097 * *$ \\
\hline
\end{tabular}

Anmerkung: $* * p<0,010$

\subsection{Mangel an Zuwendung als Risikofaktor}

Bei der bisherigen Betrachtung von Scheidung/Trennung der Eltern als Risikofaktor für geringere Lebenszufriedenheit konnte gezeigt werden, dass der Effekt der Scheidung/Trennung (distaler Risikofaktor) vollständig über die elterliche Zuwendung erklärt werden kann und somit ein Zuwendungsdefizit als eigentlicher (proximaler) Risikofaktor zu betrachten ist. In weiterer Folge soll daher geklärt werden, in welcher Beziehung die Schutzfaktoren Wertschätzung der eigenen Meinung und Selbstwirksamkeitserwartung mit dem Risikofaktor des Zuwendungsmangels stehen. Inwieweit können also Wertschätzung und Selbstwirksamkeit vor den negativen Folgen eines Zuwendungsmangels schützen?

\subsubsection{Mangel an Zuwendung als Risikofaktor - Wertschätzung der eigenen Meinung als Schutzfaktor}

Tab. 6 zeigt die Ergebnisse der hierarchischen Regressionsanalyse mit Zuwendung als Prädiktor für Lebenszufriedenheit unter Berücksichtigung der Wertschätzung der eigenen Meinung.

Tab. 6 Hierarchische Regressionsanalyse. Abhängige Variable: Allgemeine Lebenszufriedenheit ( $1=$ sehr zufrieden, $5=$ gar nicht zufrieden)

\begin{tabular}{llll}
\hline & Modell 1 & Modell 2 & Modell 3 \\
& $\beta$ & $\beta$ & $\beta$ \\
\hline Zuwendung (1= hoch bis 6= niedrig) & $0,387^{* *}$ & $0,290^{* *}$ & $0,244^{* *}$ \\
Wertschätzung (1= hoch bis 3 = niedrig) & - & $0,287^{* *}$ & $0,277^{* *}$ \\
Interaktion Zuwendung*Wertschätzung & - & - & $0,121^{* *}$ \\
$R^{2}$ & $0,15^{* *}$ & $0,223^{* *}$ & $0,235^{* *}$ \\
Änderung in $R^{2}$ & - & $0,073^{* *}$ & $0,012^{* *}$ \\
\hline
\end{tabular}

Anmerkung: $* * p<0,010$ 
Tab. 7 Trivariate Kreuztabelle: Abhängige Variable: Allgemeine Lebenszufriedenheit (dichotomiert). Unabhängige Variable: Zuwendung (dichotomiert). Kontrollvariable: Wertschätzung der eigenen Meinung (dichotomiert)

\begin{tabular}{|c|c|c|c|c|c|c|}
\hline \multicolumn{3}{|c|}{ Wertschätzung } & \multicolumn{2}{|l|}{ Zuwendung } & \multicolumn{2}{|l|}{ Gesamt } \\
\hline \multirow{3}{*}{$\overline{\text { Hoch }}$} & Zufriedenheit & H人 & $85 ?$ & 653 & 786 & 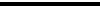 \\
\hline & & Niedrig & 14.8 & 34.7 & 214 & _ \\
\hline & Gesamt & & 100,0 & 100,0 & 100,0 & $0,229 * *$ \\
\hline \multirow[t]{3}{*}{ Gering } & Zufriedenheit & Hoch & 67,4 & 36,3 & 48,3 & - \\
\hline & & Niedrig & 32,6 & 63,7 & 51,7 & - \\
\hline & Gesamt & & 100,0 & 100,0 & 100,0 & $0,303 * *$ \\
\hline \multirow[t]{3}{*}{ Gesamt } & Zufriedenheit & Hoch & 80,7 & 50,1 & 67,3 & - \\
\hline & & Niedrig & 19,3 & 49,9 & 32,7 & - \\
\hline & Gesamt & & 100,0 & 100,0 & 100,0 & $0,323 * *$ \\
\hline
\end{tabular}

Anmerkung: $* * p<0,010$

Der Vergleich der drei Modelle zeigt, dass sowohl Zuwendung als auch Wertschätzung direkt zu einer positiven Lebenszufriedenheit beitragen, die beiden Dimensionen aber auch teilweise konfundiert sind, womit sich ein Teil des Einflusses der Zuwendung auf die Lebenszufriedenheit über die Wertschätzung erklären lässt. Ebenso liegt eine Interaktion zwischen Zuwendung und Wertschätzung vor. Das Ausmaß der Wertschätzung verändert also auch die Einflussstärke der Zuwendung auf die Lebenszufriedenheit. Die Interaktion ist in Tab. 7 wieder als trivariate Kreuztabelle dargestellt.

Wiederum ist zu erkennen, dass der Zusammenhang zwischen elterlicher Zuwendung und Lebenszufriedenheit der Kinder bei hoher Wertschätzung niedriger ausfällt als bei geringerer Wertschätzung. Somit liegen bei dieser Konstellation sowohl ein additiver Effekt als auch ein Mediator- und ein Moderatoreffekt vor. Es kann gefolgert werden, dass a) Kinder, die mehr elterliche Zuwendung erfahren, sich insgesamt auch mehr in ihrer Meinung wertgeschätzt fühlen, was wiederum zur Lebenszufriedenheit beiträgt. b) Der Effekt der Zuwendung auf die Lebenszufriedenheit lässt sich aber nur zum Teil über die Wertschätzung erklären. Beide Einflussfaktoren tragen auch unabhängig voneinander zur Lebenszufriedenheit bei. c) Je mehr sich Kinder in ihrer Meinung wertgeschätzt fühlen, desto weniger wirkt sich ein Mangel an Zuwendung negativ auf ihre Lebenszufriedenheit aus. Wertschätzung kann also als Schutzfaktor betrachtet werden, der das Risiko eines Zuwendungsmangels teilweise kompensieren (additiver Effekt), aber auch teilweise abpuffern (Moderatoreffekt) kann.

\subsubsection{Mangel an Zuwendung als Risikofaktor-Selbstwirksamkeitserwartung als Schutzfaktor}

Dieselbe Konstellation von Beziehung zeigt sich auch, wenn bei der Analyse der elterlichen Zuwendung als Prädiktor für Lebenszufriedenheit Selbstwirksamkeitserwartung anstelle von Wertschätzung untersucht wird. Die Tab. 8 und 9 zeigen die 
Tab. 8 Hierarchische Regressionsanalyse. Abhängige Variable: Allgemeine Lebenszufriedenheit ( 1 = sehr zufrieden, 5 = gar nicht zufrieden)

\begin{tabular}{llll}
\hline & Modell 1 & Modell 2 & Modell 3 \\
& $\beta$ & $\beta$ & $\beta$ \\
\hline Zuwendung (1= hoch bis 6= niedrig) & $0,380^{* *}$ & $0,283^{* *}$ & $0,264^{* *}$ \\
Selbstwirksamkeit & - & $0,389^{* *}$ & $0,379^{* *}$ \\
$(9=$ hoch bis 36= niedrig) & & & \\
Interaktion Zuwendung*Selbstwirksamkeit & - & - & $0,083^{* *}$ \\
$R^{2}$ & $0,144^{* *}$ & $0,286^{* *}$ & $0,293^{* *}$ \\
Änderung in $R^{2}$ & - & $0,142^{* *}$ & $0,006^{* *}$ \\
\hline
\end{tabular}

Anmerkung: $* * p<0,010$

Tab. 9 Trivariate Kreuztabelle: Abhängige Variable: Allgemeine Lebenszufriedenheit (dichotomiert). Unabhängige Variable: Zuwendung (dichotomiert). Kontrollvariable: Selbstwirksamkeitserwartung (dichotomiert)

\begin{tabular}{|c|c|c|c|c|c|c|}
\hline \multicolumn{3}{|c|}{ Selbstwirksamkeit } & \multirow{2}{*}{$\begin{array}{l}\text { Zuwendung } \\
\text { Hoch }(\%) \\
88,2\end{array}$} & \multirow{2}{*}{$\frac{\text { Niedrig }(\%)}{71,6}$} & $\begin{array}{l}\text { Gesamt } \\
(\%)\end{array}$ & \multirow{2}{*}{$\frac{\text { Phi }}{-}$} \\
\hline Hoch & Zufriedenheit & Hoch & & & 82,2 & \\
\hline & & Niedrig & 11,8 & 28,4 & 17,8 & - \\
\hline & Gesamt & & 100,0 & 100,0 & 100,0 & $0,208 * *$ \\
\hline \multirow[t]{3}{*}{ Gering } & Zufriedenheit & Hoch & 69,5 & 34,8 & 50,9 & - \\
\hline & & Niedrig & 30,5 & 65,2 & 49,1 & - \\
\hline & Gesamt & & 100,0 & 100,0 & 100,0 & $0,346^{* *}$ \\
\hline \multirow[t]{3}{*}{ Gesamt } & Zufriedenheit & Hoch & 80,5 & 49,9 & 66,8 & - \\
\hline & & Niedrig & 19,5 & 50,1 & 33,2 & - \\
\hline & Gesamt & & 100,0 & 100,0 & 100,0 & $0,324 * *$ \\
\hline
\end{tabular}

Anmerkung: $* * p<0,010$

Ergebnisse der Regressionsanalysen und veranschaulichen die Richtung der Interaktion.

Auch hier weisen die Ergebnisse darauf hin, dass ein Teil des Einflusses der Zuwendung auf die Lebenszufriedenheit über eine höhere Selbstwirksamkeit erklärt werden kann, jedoch sowohl Zuwendung als auch Selbstwirksamkeit unabhängig voneinander zu höherer Lebenszufriedenheit beitragen. Darüber hinaus liegt

Tab. 10 Hierarchische Regressionsanalyse. Abhängige Variable: Allgemeine Lebenszufriedenheit ( $1=$ sehr zufrieden, $5=$ gar nicht zufrieden)

\begin{tabular}{llll}
\hline & Modell 1 & Modell 2 & Modell 3 \\
& $\beta$ & $\beta$ & $\beta$ \\
\hline Wertschätzung (1= hoch bis 3 = niedrig) & $0,378^{* *}$ & $0,255^{* *}$ & $0,239^{* *}$ \\
Selbstwirksamkeit & - & $0,378^{* *}$ & $0,343^{* *}$ \\
(9= hoch bis 36= niedrig) & & & \\
Interaktion Wertschätzung*Selbstwirksamkeit & - & - & $0,145^{* *}$ \\
$R^{2}$ & $0,143^{* *}$ & $0,271^{* *}$ & $0,29 * *$ \\
Änderung in $R^{2}$ & - & $0,128^{* *}$ & $0,019^{* *}$ \\
\hline
\end{tabular}

Anmerkung: ** $p<0,010$ 
Tab. 11 Trivariate Kreuztabelle: Abhängige Variable: Allgemeine Lebenszufriedenheit (dichotomiert). Unabhängige Variable: Wertschätzung der eigenen Meinung (dichotomiert). Kontrollvariable: Selbstwirksamkeitserwartung (dichotomiert)

\begin{tabular}{|c|c|c|c|c|c|c|}
\hline \multicolumn{3}{|c|}{ Selbstwirksamkeit } & \multicolumn{2}{|c|}{ Wertschätzung } & \multicolumn{2}{|c|}{ Gesamt } \\
\hline & & & Hoch (\%) & Niedrig (\%) & & FIII \\
\hline \multirow[t]{3}{*}{ Hoch } & Zufriedenheit & Hoch & 88,3 & 67,7 & 82,4 & - \\
\hline & & Niedrig & 11,7 & 32,3 & 17,6 & - \\
\hline & Gesamt & & 100,0 & 100,0 & 100,0 & $0,243 * *$ \\
\hline \multirow[t]{3}{*}{ Gering } & Zufriedenheit & Hoch & 64,3 & 36,5 & 51,4 & - \\
\hline & & Niedrig & 35,7 & 63,5 & 48,6 & - \\
\hline & Gesamt & & 100,0 & 100,0 & 100,0 & $0,278 * *$ \\
\hline \multirow[t]{3}{*}{ Gesamt } & Zufriedenheit & Hoch & 78,2 & 48,7 & 67,2 & - \\
\hline & & Niedrig & 21,8 & 51,3 & 32,8 & - \\
\hline & Gesamt & & 100,0 & 100,0 & 100,0 & $0,305^{* *}$ \\
\hline
\end{tabular}

Anmerkung: $* * p<0,010$

ein Moderatoreffekt vor, der darauf hinweist, dass die negative Auswirkung eines Zuwendungsmangels auf die Lebenszufriedenheit mit zunehmender Selbstwirksamkeitserwartung abnimmt. Damit zeigt auch Selbstwirksamkeit als Schutzfaktor in dieser Konstellation sowohl eine kompensierende als auch eine abpuffernde Wirkung. Dies gilt auch, wenn Selbstwirksamkeit als Schutzfaktor im Zusammenhang zwischen Wertschätzung und Lebenszufriedenheit untersucht wird (Tab. 10 und 11). Ein Teil des negativen Einflusses mangelnder Wertschätzung auf die Lebenszufriedenheit kann über geringere Selbstwirksamkeitserwartung erklärt werden, jedoch tragen Wertschätzung und Selbstwirksamkeit auch unabhängig voneinander zur Lebenszufriedenheit bei. Bei Kindern mit höherer Selbstwirksamkeitserwartung wirkt sich ein Mangel an Wertschätzung aber weniger beeinträchtigend auf die Lebenszufriedenheit aus. Somit wirkt Selbstwirksamkeit auch hier sowohl kompensierend als auch abpuffernd.

\subsection{Armutserfahrung und Lebenszufriedenheit}

\subsubsection{Elterliche Zuwendung als Schutzfaktor}

Nach derselben Analysestrategie wurden die Merkmale elterliche Zuwendung, Wertschätzung der eigenen Meinung und Selbstwirksamkeitserwartung auch als Schutzfaktoren beim Zusammenhang von Armutserfahrung und Lebenszufriedenheit untersucht. Tab. 12 zeigt die Ergebnisse der hierarchischen Regressionsanalyse mit Zuwendung als potenziellem Schutzfaktor.

Es wird ersichtlich, dass Armutserfahrungen mit geringerer Lebenszufriedenheit verbunden sind. Ein kleiner Teil des Armutseffekts kann über einen Mangel an Zuwendung erklärt werden. Somit beinhalten Armutserfahrungen bis zu einem gewissen Grad auch das Risiko, weniger elterliche Zuwendung zu erfahren. Neben dem positiven Effekt der Zuwendung bleibt aber ein beeinträchtigender Effekt der Armutserfahrung bestehen. Darüber hinaus lässt sich kein Moderatoreffekt nachwei- 
Tab. 12 Hierarchische Regressionsanalyse. Abhängige Variable: Allgemeine Lebenszufriedenheit ( 1 = sehr zufrieden, 5 = gar nicht zufrieden)

\begin{tabular}{llll}
\hline & Modell 1 & Modell 2 & Modell 3 \\
& $\beta$ & $\beta$ & $\beta$ \\
\hline Armutserfahrungen (1= nein, 2=ja) & $0,167^{* *}$ & $0,094^{* *}$ & $0,087^{* *}$ \\
Zuwendung (1 = hoch bis 6= niedrig) & - & $0,365^{* *}$ & $0,361^{* *}$ \\
Interaktion Armutserfahrung*Zuwendung & - & - & 0,022 \\
$R^{2}$ & $0,028^{* *}$ & $0,156^{* *}$ & $0,156^{* *}$ \\
Änderung in $R^{2}$ & - & $0,128^{* *}$ & 0,000 \\
\hline
\end{tabular}

Anmerkung: ** $p<0,010$

sen. Von einer Schutzwirkung kann daher nur im Sinne eines weiteren förderlichen Faktors bzw. einer Kompensation (additiver Effekt) gesprochen werden.

\subsubsection{Wertschätzung der eigenen Meinung als Schutzfaktor}

Ähnlich ist das Bild bei der Untersuchung von wahrgenommener Wertschätzung der eigenen Meinung als Schutzfaktor (siehe Tab. 13). Die Konfundierung zwischen Armutserfahrung und Wertschätzung ist vernachlässigbar gering. Armutserfahrung und Wertschätzung tragen unabhängig voneinander zur Erklärung von Lebenszufriedenheit bei. Eine Interaktion der beiden Variablen lässt sich nicht nachweisen. Somit liegt kein Moderatoreffekt vor, der die Wirkung der Armutserfahrung abpuffern würde. Wertschätzung kann hier nur als eigenständiger Einflussfaktor kompensierend (additiver Effekt) wirken.

\subsubsection{Selbstwirksamkeitserwartung als Schutzfaktor}

In Tab. 14 wird schließlich der Einfluss der Selbstwirksamkeitserwartung auf den Zusammenhang zwischen Armutserfahrung und Lebenszufriedenheit untersucht. Im Vergleich zur zuvor untersuchten Zuwendung und Wertschätzung weist Selbstwirksamkeit eine etwas höhere Konfundierung mit Armutserfahrung auf. Das bedeutet, dass ein Teil des Effekts von Armutserfahrung auf die Lebenszufriedenheit über eine geringere Selbstwirksamkeitserwartung erklärt werden kann. Kinder mit Armutserfahrungen haben weniger das Gefühl, Einfluss auf ihr eigenes Leben nehmen zu können und sind dadurch weniger zufrieden mit ihrem Leben. Der Effekt der

Tab. 13 Hierarchische Regressionsanalyse. Abhängige Variable: Allgemeine Lebenszufriedenheit ( $1=$ sehr zufrieden, 5 = gar nicht zufrieden)

\begin{tabular}{llll}
\hline & Modell 1 & Modell 2 & Modell 3 \\
& $\beta$ & $\beta$ & $\beta$ \\
\hline Armutserfahrungen $(1=$ nein, 2=ja) & $0,163^{* *}$ & $0,124^{* *}$ & $0,114^{* *}$ \\
Wertschätzung $(1=$ hoch bis 3= niedrig) & - & $0,376^{* *}$ & $0,373^{* *}$ \\
Interaktion Armutserfahrung*Wertschätzung & - & - & 0,047 \\
$R^{2}$ & $0,027^{* *}$ & $0,166^{* *}$ & $0,168^{* *}$ \\
Änderung in $R^{2}$ & - & $0,14^{* *}$ & 0,002 \\
\hline
\end{tabular}

Anmerkung: $* * p<0,010$ 
Tab. 14 Hierarchische Regressionsanalyse. Abhängige Variable: Allgemeine Lebenszufriedenheit ( $1=$ sehr zufrieden, $5=$ gar nicht zufrieden)

\begin{tabular}{llll}
\hline & Modell 1 & Modell 2 & Modell 3 \\
& $\beta$ & $\beta$ & $\beta$ \\
\hline Armutserfahrungen (1= nein, 2=ja) & $0,160^{* *}$ & $0,070^{* *}$ & $0,075^{* *}$ \\
Selbstwirksamkeit & - & $0,471^{* *}$ & $0,473^{* *}$ \\
9= hoch bis 36= niedrig) & & & \\
Interaktion Armutserfahrung*Selbstwirksamkeit & - & - & $-0,015$ \\
$R^{2}$ & $0,026^{* *}$ & $0,239^{* *}$ & $0,239^{* *}$ \\
Änderung in $R^{2}$ & - & 0,213 & 0,000 \\
\hline
\end{tabular}

Anmerkung: ** $p<0,010$

Armutserfahrung wird aber wiederum nicht vollständig durch die Berücksichtigung der Selbstwirksamkeit aufgelöst. Somit tragen beide Merkmale auch unabhängig voneinander zur Erklärung von Lebenszufriedenheit bei. Eine Interaktion der beiden Merkmale und somit ein Moderatoreffekt sind wiederum nicht nachzuweisen. Somit zeigt Selbstwirksamkeit in diesem Zusammenhang lediglich kompensierende Wirkung als eigenständiger Einflussfaktor auf Lebenszufriedenheit (additiver Effekt), jedoch keine abpuffernde Wirkung, die den Einfluss der Armutserfahrung auf die Lebenszufriedenheit abschwächen würde.

\section{Zusammenfassung, Diskussion und Untersuchungsbegrenzungen}

Die vorliegende Studie untersuchte anhand der Daten eines Kindersurveys das Resilienzphänomen als relationales Konzept und betrachtete, wie potenzielle Schutzfaktoren (elterliche Zuwendung, Wertschätzung der eigenen Meinung und Selbstwirksamkeitserwartung) den Zusammenhang zwischen Risikofaktoren (Trennung/ Scheidung der Eltern und Armutserfahrungen) und Lebenszufriedenheit als Outcomevariable beeinflussen. Das Augenmerk lag dabei auf dem Vorhandensein von additiven Effekten, Mediatoreffekten und Moderatoreffekten, die als unterschiedliche Erscheinungsformen des Resilienzphänomens betrachtet werden können. Es zeigte sich, dass der Risikofaktor einer Trennung oder Scheidung der Eltern mit einer geringeren Lebenszufriedenheit bei Kindern verbunden ist. Wenn Kinder jedoch ausreichend Zuwendung von den Eltern bekommen, dann lässt sich keine Auswirkung der Trennung oder Scheidung auf die Lebenszufriedenheit der Kinder mehr feststellen. Statistisch gesprochen wird der Effekt der Trennung bzw. Scheidung durch die elterliche Zuwendung also vollständig konfundiert. Somit kann die Trennung der Eltern als distaler Risikofaktor betrachtetet werden, dessen beeinträchtigende Wirkung auf die Lebenszufriedenheit durch einen Mangel an Zuwendung (als proximalen Risikofaktor) indirekt vermittelt wird.

Für die Lebenszufriedenheit ist also die elterliche Zuwendung entscheidend. Eine Trennung/Scheidung der Eltern erhöht lediglich das Risiko, weniger elterliche Zuwendung zu erfahren. Anders ausgedrückt: Liegt ein Mangel an Zuwendung vor, spielt es keine Rolle, ob die Eltern getrennt sind oder zusammenleben. Es wirkt sich nachteilig auf die Lebenszufriedenheit aus, ebenso wie sich ausreichende Zuwen- 
dung positiv auswirkt, egal ob Eltern getrennt sind oder zusammenleben. Lediglich ist ein Mangel an Zuwendung unter Kindern mit getrennten oder geschiedenen Eltern häufiger anzutreffen. Es bleibt eine Frage der (nicht einheitlichen) Definition, ob glückliche Scheidungskinder, die ausreichend elterliche Zuwendung erfahren, als resilient bezeichnet werden können oder, wie Lösel und Bender (2007, S. 63) bemerken, sie einfach einem geringeren Risiko ausgesetzt waren als Kinder, die zusätzlich zur Scheidung/Trennung der Eltern proximalen Risikofaktoren wie einem Zuwendungsdefizit ausgesetzt sind.

Wahrgenommene Wertschätzung der eigenen Meinung sowie Selbstwirksamkeitserwartung zeigten hingegen neben additiven Effekten auch moderierenden Einfluss auf den Zusammenhang zwischen Trennung/Scheidung der Eltern und Lebenszufriedenheit. Beide Faktoren wirken also protektiv sowohl im kompensatorischen Sinne (additiver Effekt) als auch im Sinne eines Abpuffereffekts (Moderation). Kompensatorisch insofern, dass höhere Wertschätzung oder Selbstwirksamkeit sich unabhängig vom weiterhin vorhandenen Trennungs-/Scheidungseffekt positiv auf die Lebenszufriedenheit auswirkt. Inwieweit dies bereits als Resilienzphänomen anzusehen ist, ist wiederum Definitionssache und wird von einigen AutorInnen kritisch gesehen (z.B. Laucht 1999; Masten 2001; Rutter 1990). Was den gängigen Vorstellungen von Resilienz jedoch am nächsten kommt, ist die abpuffernde Wirkung durch den beobachteten Moderatoreffekt. Mehr Wertschätzung oder höhere Selbstwirksamkeit vermindern den beeinträchtigenden Effekt einer Trennung oder Scheidung auf die Lebenszufriedenheit. Anders ausgedrückt: Bei Kindern, die sich in ihrer Meinung wenig wertgeschätzt fühlen oder weniger das Gefühl haben, Einfluss auf ihr Leben nehmen zu können, spielt eine Trennung der Eltern für die Lebenszufriedenheit eine Rolle. Bei Kindern mit hoher wahrgenommener Wertschätzung oder hoher Selbstwirksamkeit spielt eine Trennung keine oder kaum eine Rolle. Dasselbe Bild bestätigt sich, wenn anstelle einer Trennung/Scheidung der Eltern (distaler Faktor) die wahrgenommene elterliche Zuwendung (proximaler Faktor) als Risiko für die Beeinträchtigung der Lebenszufriedenheit untersucht wird. Neben additiver und vermittelnder Wirkung (Mediation) konnte auch hier ein deutlicher Moderatoreffekt nachgewiesen werden. Ein Mangel an Zuwendung spielt also dann eine Rolle, wenn die Schutzfaktoren Wertschätzung oder Selbstwirksamkeitserwartung nicht oder nur in geringem Ausmaß vorhanden sind. Beim Vorhandensein der Schutzfaktoren verliert der Zuwendungsmangel hingegen seine Bedeutung für die Lebenszufriedenheit. Dieselbe Wirkung zeigt die Selbstwirksamkeitserwartung beim Zusammenhang zwischen Wertschätzung und Lebenszufriedenheit. Ein Mangel an wahrgenommener Wertschätzung ist dann bedeutend für die Lebenszufriedenheit, wenn die Selbstwirksamkeitserwartung gering ist. Bei höherer Selbstwirksamkeit verliert der Effekt seine Bedeutung.

Ein anderes Bild ergibt sich bei der Untersuchung von Armutserfahrung als Risikofaktor. Armutserfahrung ist mit geringerer Lebenszufriedenheit verbunden. Die untersuchten potenziellen Schutzfaktoren Zuwendung, Wertschätzung und Selbstwirksamkeitserwartung haben zwar einen additiven und indirekt vermittelnden Einfluss (Mediation) auf die Lebenszufriedenheit, jedoch kann kein moderierender Einfluss nachgewiesen werden. Eine protektive Wirkung zeigt sich also nur im kompensatorischen Sinne, indem die Schutzfaktoren für sich unabhängig vom Risiko der 
Armutserfahrung positiv zur Lebenszufriedenheit beitragen. Ein beeinträchtigender Effekt der Armutserfahrung bleibt aber weiterhin bestehen.

Gesamt betrachtet zeigt sich, dass in verschiedenen Konstellationen von möglichen Risiko- und Schutzfaktoren sowohl additive Effekte als auch Mediator- und Moderatoreffekte zu finden sind. Dies untermauert den variablen, situationsspezifischen und multidimensionalen Charakter des Resilienzbegriffs und das Zutreffen von unterschiedlichen Resilienzmodellen in verschiedenen Kontexten. Mit Blick auf die allgemeine Lebenszufriedenheit von Kindern erweisen sich die wahrgenommene Wertschätzung der eigenen Meinung sowie die Selbstwirksamkeitserwartung als protektive Faktoren gegen das Risiko einer elterlichen Trennung/Scheidung und damit einhergehend einem Mangel an elterlicher Zuwendung, indem sie imstande sind, den beeinträchtigenden Einfluss zu verringern. Nicht festgestellt werden konnte Gleiches für den Risikofaktor Armutserfahrung. Wertschätzung und Selbstwirksamkeit wirken zwar kompensierend, indem sie eigenständig zur Lebenszufriedenheit beitragen, jedoch bleibt der Einfluss der Armutserfahrung auch bei hoher Wertschätzung und Selbstwirksamkeit vorhanden.

Die Ergebnisse dieser Studie zeigen deutlich, dass nicht von Resilienz im Allgemeinen gesprochen werden kann, sondern die unterschiedlichen Konstellationen von Bedingungen, unter denen Kinder heranwachsen, spezifisch betrachtet werden müssen. Diese Studie gibt Hinweise darauf, wie verschiedene Wirkungszusammenhänge zwischen Risiko- und Schutzfaktoren aussehen können und trägt somit zu einem besseren Verständnis der diversen Formen bei, wie sich das mit dem Begriff Resilienz beschriebene Phänomen empirisch äußert. Über die Identifikation von Schutzfaktoren und die Beschreibung ihrer unterschiedlichen Wirkungszusammenhänge hinaus bleibt die Entwicklung einer Theorie oder erklärender Ansätze für das Zustandekommen von Resilienz allerdings weiterhin ein Desiderat (vgl. Wustmann 2004, 2005), welchem mit quantitativ ausgerichteten Querschnittsstudien nicht beigekommen werden kann. Die Resilienzforschung ist daher auch gefordert, der Komplexität des Phänomens und seiner Entstehung durch eine qualitativ ausgerichtete Perspektive gerecht zu werden. Beispielsweise plädiert Gabriel (2005, S. 212) für Fallstudien, die das Augenmerk auf biografische Übergänge (Transitionen) legen, um so resiliente Bewältigungsmuster rekonstruieren zu können. Fruchtvoll erschiene auch eine Kombination qualitativer und quantitativer Ansätze, die auf typenbildende Aggregationen abzielen. Hier wäre es beispielsweise möglich, mittels Clusteranalysen anhand repräsentativer Stichproben Differenzlinien auszumachen und ähnliche Fälle zu gruppieren. Diese Typen könnten dann mittels tiefgehender qualitativer Fallstudien untersucht werden (für ein Beispiel zur qualitativen Bildung von Resilienztypen auf Haushaltsebene siehe Promberger 2017).

Eine weitere Untersuchungsbegrenzung liegt darin, dass die vorliegende Studie bereits vorhandene Daten, deren Erhebungszweck nicht primär die Beantwortung der hier aufgeworfenen Fragestellungen war, analysiert. Dementsprechend musste sich die Auswahl und Operationalisierung der untersuchten Variablen nach den vorhandenen Möglichkeiten richten. Natürlich wären Untersuchungen zu weiteren Risikound Schutzfaktoren sowie weiteren Outcomevariablen in verschiedenen Konstellationen von Interesse. Des Weiteren ist zu beachten, dass die Daten aus einer Querschnittserhebung stammen, die eine empirische Erfassung des Resilienzphänomens 
nur im Sinne des Ergebnisses erfolgreicher Adaption angesichts herausfordernder Umstände erlauben, während ein breiterer Resilienzbegriff aber nicht nur das Ergebnis, sondern auch den Prozess und die Fähigkeit erfolgreicher Adaption umfasst. Um dem prozesshaften Charakter von Resilienz gerecht zu werden, sind Studien im Längsschnittdesign unerlässlich (vgl. Wustmann 2004, S. 53).

Danksagung Die AutorInnen bedanken sich bei Franz Höllinger für Kommentare und Anmerkungen zu einer frühen Fassung des Beitrags.

Funding Open access funding provided by University of Graz.

Open Access Dieser Artikel wird unter der Creative Commons Namensnennung 4.0 International Lizenz veröffentlicht, welche die Nutzung, Vervielfältigung, Bearbeitung, Verbreitung und Wiedergabe in jeglichem Medium und Format erlaubt, sofern Sie den/die ursprünglichen Autor(en) und die Quelle ordnungsgemäß nennen, einen Link zur Creative Commons Lizenz beifügen und angeben, ob Änderungen vorgenommen wurden.

Die in diesem Artikel enthaltenen Bilder und sonstiges Drittmaterial unterliegen ebenfalls der genannten Creative Commons Lizenz, sofern sich aus der Abbildungslegende nichts anderes ergibt. Sofern das betreffende Material nicht unter der genannten Creative Commons Lizenz steht und die betreffende Handlung nicht nach gesetzlichen Vorschriften erlaubt ist, ist für die oben aufgeführten Weiterverwendungen des Materials die Einwilligung des jeweiligen Rechteinhabers einzuholen.

Weitere Details zur Lizenz entnehmen Sie bitte der Lizenzinformation auf http://creativecommons.org/ licenses/by/4.0/deed.de.

\section{Literatur}

Aiken, Leona S., und Stephen G. West. 1991. Multiple regression: testing and interpreting interactions. Newbury Park: SAGE.

Antonovsky, Aaron. 1987. Unraveling the mystery of health. How people manage stress and stay well. San Francisco: Jossey-Bass.

Bandura, Albert. 1997. Self-efficacy. The exercise of control. New York: W H Freeman, Times Books, Henry Holt \& Co.

Block, Jeanne H., und Jack Block. 1980. The role of ego-control and ego-resilience in the organization of behavior. In Development of cognition, affect and social relations, Hrsg. Andrew Collins, 39-101. New York: Psychology Press.

Bock, Karin. 2010. Kinderalltag - Kinderwelten. Rekonstruktive Analysen von Gruppendiskussionen mit Kindern. Opladen: Budrich.

Bonß, Wolfgang. 2015. Karriere und sozialwissenschaftliche Potenziale des Resilienzbegriffs. In Resilienz im Sozialen. Theoretische und empirische Analysen, Hrsg. Martin Endreß, Andrea Maurer, 15-31. Wiesbaden: Springer VS.

Bürkner, Hans-Joachim. 2010. Vulnerabilität und Resilienz - Forschungsstand sozialwissenschaftlicher Untersuchungsperspektiven. Working Paper, Erkner, Leibniz-Institut für Regionalentwicklung und Strukturplanung. https://leibniz-irs.de/fileadmin/user_upload/IRS_Working_Paper/wp_vr.pdf. Zugegriffen: 21. Okt. 2019.

Dür, Wolfgang, und Robert Griebler. 2007. Die Gesundheit der österreichischen SchülerInnen im Lebenszusammenhang. Ergebnisse des 7. WHO-HBSC-Surveys 2006. Wien: Bundesministerium für Gesundheit, Familie und Jugend.

Fingerle, Michael. 1999. Resilienz - Vorhersage und Förderung. In Was Kinder stärkt: Erziehung zwischen Risiko und Resilienz, 1. Aufl., Hrsg. Günther Opp, Michael Fingerle, und Andreas Freytag, 94-98. München: Ernst Reinhardt Verlag.

Fingerle, Michael. 2007. Der „riskante“ Begriff der Resilienz - Überlegungen zur Resilienzförderung im Sinne der Organisation von Passungsverhältnissen. In Was Kinder stärkt - Erziehung zwischen Risiko und Resilienz, 2. Aufl., Hrsg. Günther Opp, Michael Fingerle, 299-310. München: Ernst Reinhardt Verlag. 
Friedrich, Monika. 2012. Das Zauberwort heißt Resilienz - Eine Analyse zum Resilienzkonzept. In Beziehungsweise - Informationsdienst des österreichischen Instituts für Familienforschung.

Gabriel, Thomas. 2005. Resilienz - Kritik und Perspektiven. Zeitschrift für Pädagogik 51(2):207-217.

Garmezy, Norman. 1983. Stressors of childhood. In Stress, coping, and development in children, Hrsg. Norman Garmezy, Michael Rutter, 43-84. New York: McGraw-Hill.

Hurrelmann, Klaus, und Sabine Andresen. 2010. Kinder in Deutschland 2010. 2. World Vision Kinderstudie. Frankfurt a. Main: S. Fischer.

Jäkel, Julia, Dieter Wolke, und Birgit Leyendecker. 2012. Resilienz im Vorschulalter: Wie stark kann die familiäre Leseumwelt biologische und soziokulturelle Entwicklungsrisiken kompensieren? Zeitschrift für Familienforschung 24:148-159.

Keck, Markus, und Patrick Sakdapolrak. 2013. What is social resilience? Lessons learned and ways forward. Erdkunde 67:5-18. https://doi.org/10.3112/erdkunde.2013.01.02.

Kobasa, Suzanne C. 1982. The hardy personality: toward a social psychology of stress and health. In Social psychology of health and illness, Hrsg. Glenn S. Sanders, Jerry Suls, 3-32. Hillsdale: Lawrence Erlbaum.

Kühnel, Steffen, und Dagmar Krebs. 2001. Statistik für Sozialwissenschaften. Grundlagen, Methoden, Anwendungen, 2. Aufl., Reinbek: Rowohlt.

Laucht, Manfred. 1999. Risiko- vs. Schutzfaktor? Kritische Anmerkungen zu einer problematischen Dichotomie. In Was Kinder stärkt: Erziehung zwischen Risiko und Resilienz, 1. Aufl., Hrsg. Günther Opp, Michael Fingerle, und Andreas Freytag, 303-314. München: Ernst Reinhardt Verlag.

Leipold, Bernard, und Werner Greve. 2009. Resilience. A conceptual bridge between coping and development. European Psychologist 14:40-50.

Leppert, Karena, Benjamin Koch, Elmar Brähler, und Bernhard Strauß. 2008. Die Resilienzskala (RS) Überprüfung der Langform RS-25 und einer Kurzform RS-13. Klinische Diagnostik und Evaluation 2:226-243.

Lösel, Friedrich, und Doris Bender. 2007. Von generellen Schutzfaktoren zu spezifischen protektiven Prozessen: Konzeptuelle Grundlagen und Ergebnisse der Resilienzforschung. In Was Kinder stärkt: Erziehung zwischen Risiko und Resilienz, 2. Aufl., Hrsg. Günther Opp, Michael Fingerle, 57-78. München: Ernst Reinhardt Verlag.

Luthar, Suniya S., und Gretta Cushing. 1999. Measurement issues in the empirical study of resilience. An overview. In Resilience and development: Positive life adaptations, Hrsg. Meyer D. Glanz, Jeannette L. Johnson, 129-160. Dordrecht: Kluwer Academic Publishers.

Masten, Ann S. 2001. Ordinary magic: resilience processes in development. American Psychologist 56(3):227-238.

Masten, Ann S., Karin M. Best, und Norman Garmezy. 1990. Resilience and development: contributions from the study of children who overcome adversity. Development and psychopathology 2:425-444.

Promberger, Markus. 2017. Resilience among vulnerable households in Europe. Questions, concept, findings and implications. IAB-Discussion Paper 12/2017.

Promberger, Markus, Lars Meier, Frank Sowa, und Marie Boost. 2015. Chancen des Resilienzbegriffs für eine soziologische Armutsforschung. In Resilienz im Sozialen. Theoretische und empirische Analysen, Hrsg. Martin Endres, Andrea Maurer, 265-294. Wiesbaden: Springer VS.

Rutter, Michael. 1985. Resilience in the face of adversity: protective factors and resistance to psychiatric disorder. British Journal of Psychiatry 147:598-611.

Rutter, Michael. 1990. Psychosocial resilience and protective mechanisms. In Risk and protective factors in the development of psychopathology, Hrsg. Jon Ann S. Rolf Masten, Dante Cicchetti, Keith H. Nuechterlein, und Sheldon Weintraub, 181-214. Cambridge: University Press.

Schumacher, Jörg, Karena Leppert, Thomas Gunzelmann, Bernhard Strauß, und Elmar Brähler. 2005. Die Resilienzskala - Ein Fragebogen zur Erfassung der psychischen Widerstandsfähigkeit als Personenmerkmal. Zeitschrift für Klinische Psychologie, Psychiatrie und Psychotherapie 53:16-39.

Staudinger, Ursula M. 1999. Perspektiven der Resilienzforschung aus der Sicht der Lebensspannen-Psychologie. In Was Kinder stärkt: Erziehung zwischen Risiko und Resilienz, 1. Aufl., Hrsg. Günther Opp, Michael Fingerle, und Andreas Freytag, 343-350. München: Ernst Reinhardt Verlag.

Wagnild, Gail M., und Heather M. Young. 1993. Development and psychometric evaluation of the Resilience Scale. Journal of Nursing Measurement 1(2):165-178.

Werner, Emmy E., und Ruth S. Smith. 1982. Vulnerable but invincible: a longitudinal study of resilient children and youth. New York: McGraw-Hill.

Wilk, Liselotte, und Johann Bacher. 1994. Kindliche Lebenswelten. Eine sozialwissenschaftliche Annäherung. Opladen: VS. 
Wustmann, Corina. 2004. Resilienz. Widerstandsfähigkeit von Kindern in Tageseinrichtungen fördern. Weinheim: Beltz.

Wustmann, Corina. 2005. Die Blickrichtung der neueren Resilienzforschung. Wie Kinder Lebensbelastungen bewältigen. Zeitschrift für Pädagogik 51(2):192-206.

Zander, Margherita. 2008. Armes Kind - starkes Kind? Die Chance der Resilienz. Wiesbaden: Springer VS.

Zander, Margherita. 2011. Handbuch Resilienzförderung. Wiesbaden: Springer VS.

Otto Bodi-Fernandez ist wissenschaftlicher Mitarbeiter von AUSSDA - The Austrian Social Science Data Archive am Center for Social Research der Universität Graz und lehrt im Bereich Methoden empirischer Sozialforschung. Er war in zahlreichen Forschungsprojekten verschiedener Disziplinen tätig. Seine Forschungsinteressen liegen in den Bereichen soziale Ungleichheit, Umwelt, Bildung und Kindheitsforschung.

Karina Fernandez ist Hochschulprofessorin für Bildungssoziologie und Qualitätsmanagement an der Pädagogischen Hochschule Steiermark. Sie lehrt und forscht zu den Themen Jugendsoziologie, soziale Ungleichheit, Bildungssoziologie und Methoden der empirischen Sozialforschung. 